
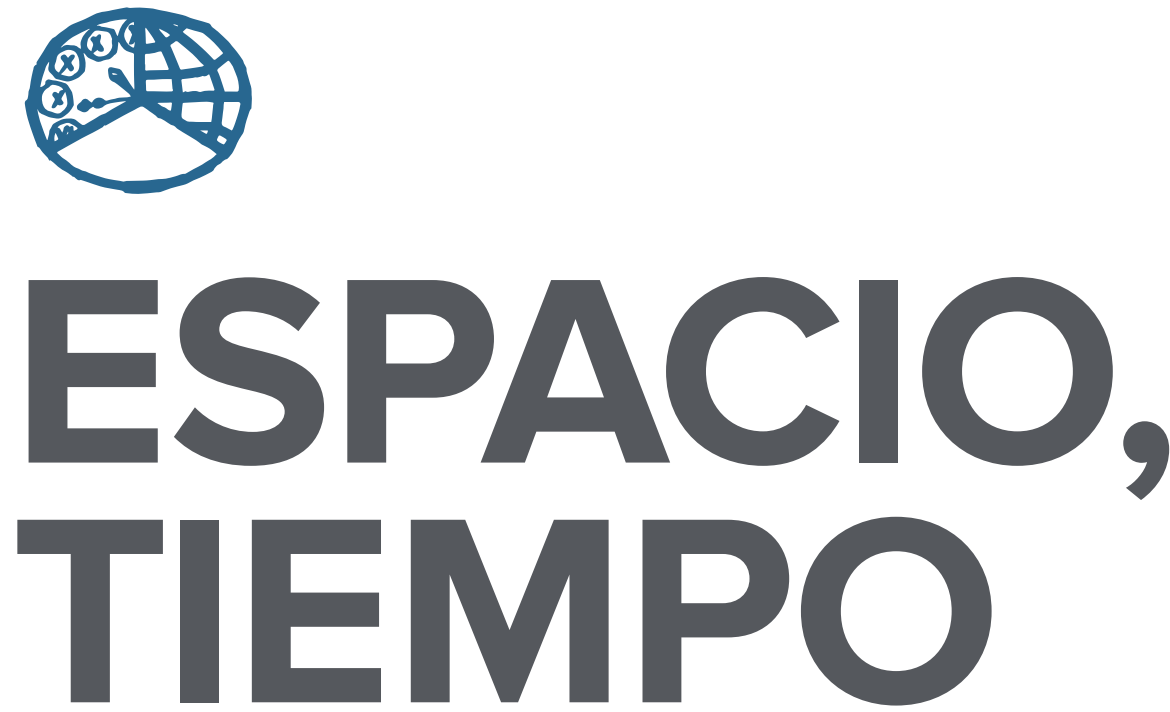

AÑO 2019 NUEVA ÉPOCA ISSN 1130-4715

E-ISSN 2340-1478
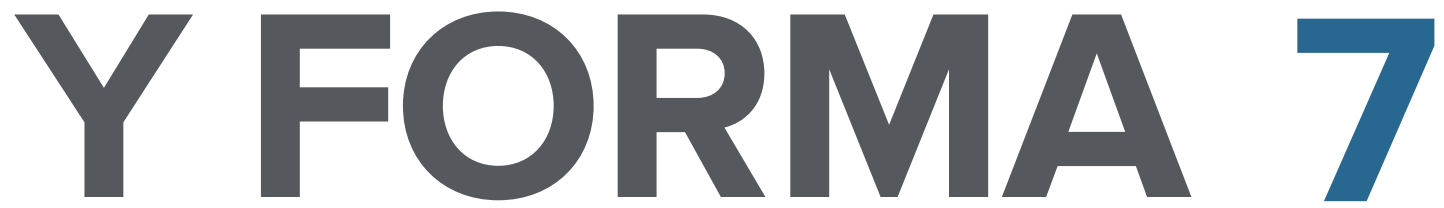

SERIE VII HISTORIA DEL ARTE

REVISTA DE LA FACULTAD DE GEOGRAFÍA E HISTORIA 


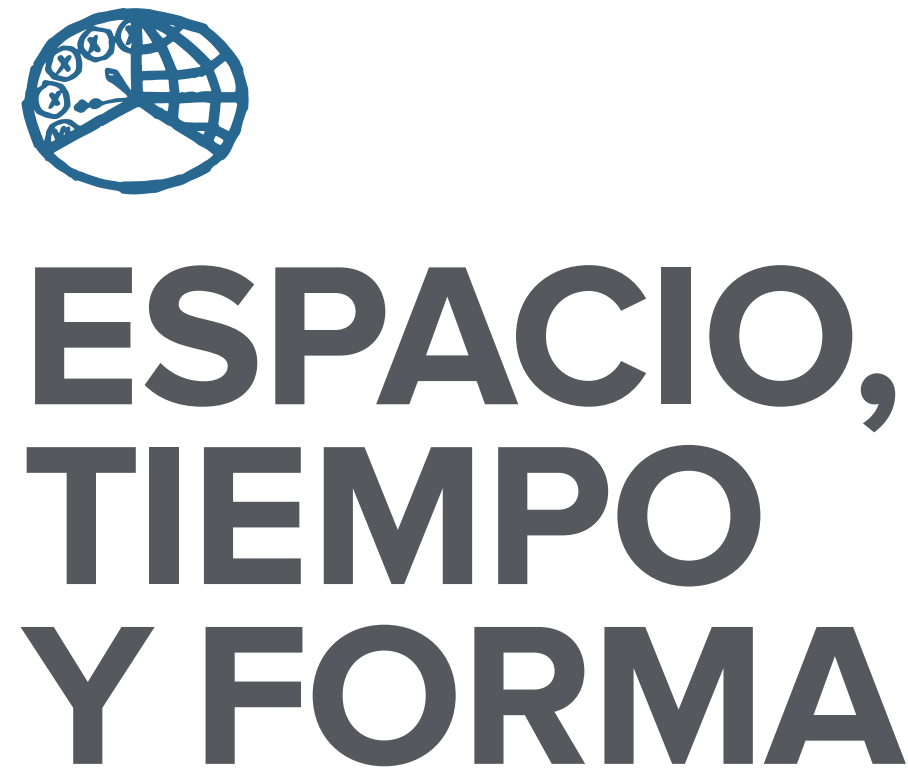

AÑO 2019

NUEVA ÉPOCA

ISSN $1130-4715$

E-ISSN 2340-1478

SERIE VII HISTORIA DEL ARTE

REVISTA DE LA FACULTAD DE GEOGRAFİA E HISTORIA

DOI: http://dx.doi.org/10.5944/etfvii.7.2019

\section{UกED}

UNIVERSIDAD NACIONAL DE EDUCACIÓN A DISTANCIA 
La revista Espacio, Tiempo y Forma (siglas recomendadas: ETF), de la Facultad de Geografía e Historia de la UNED, que inició su publicación el año 1988, está organizada de la siguiente forma:

$$
\begin{aligned}
& \text { SERIE I - Prehistoria y Arqueología } \\
& \text { SERIE II - Historia Antigua } \\
& \text { SERIE III - Historia Medieval } \\
& \text { SERIE IV - Historia Moderna } \\
& \text { SERIE V - Historia Contemporánea } \\
& \text { SERIE VI - Geografía } \\
& \text { SERIE VII - Historia del Arte }
\end{aligned}
$$

Excepcionalmente, algunos volúmenes del año 1988 atienden a la siguiente numeración:

$$
\begin{aligned}
& \mathrm{N}^{\circ} 1 \text { - Historia Contemporánea } \\
& \mathrm{N}^{\circ} 2 \text { - Historia del Arte } \\
& \mathrm{N}^{\circ} 3 \text { - Geografía } \\
& \mathrm{N} .^{\circ} 4 \text { - Historia Moderna }
\end{aligned}
$$

ETF no se solidariza necesariamente con las opiniones expresadas por los autores.

UNIVERSIDAD NACIONAL DE EDUCACIÓN A DISTANCIA

Madrid, 2019

SERIE VII · HISTORIA DEL ARTE (NUEVA ÉPOCA) N..$^{\circ} 72019$

ISSN $1130-4715 \cdot$ E-ISSN 2340-1478

DEPÓSITO LEGAL

$M-21.037-1988$

URL

ETF VII · HISTORIA DEL ARTE $\cdot$ http://revistas.uned.es/index.php/ETFVII

DISEÑO Y COMPOSICIÓN

Carmen Chincoa Gallardo · http://www.laurisilva.net/cch

Impreso en España · Printed in Spain 


\section{DOSSIER}

L'APELLE VITRUVIANO: RIFLESSIONI SULLA CULTURA ARCHITETTONICA DEI PITTORI NELLA PRIMA ETÃ MODERNA

Edito da Filippo Camerota

THE VITRUVIAN APELLES: THOUGHTS ON

THE ARCHITECTURAL CULTURE OF PAINTERS

IN THE EARLY MODERN AGE

Edited by Filippo Camerota

EL APELLES VITRUVIANO: REFLEXIONES EN TORNO

A LA CULTURA ARQUITECTÓNICA DE LOS PINTORES

DE LA EDAD MODERNA

Editado por Filippo Camerota 


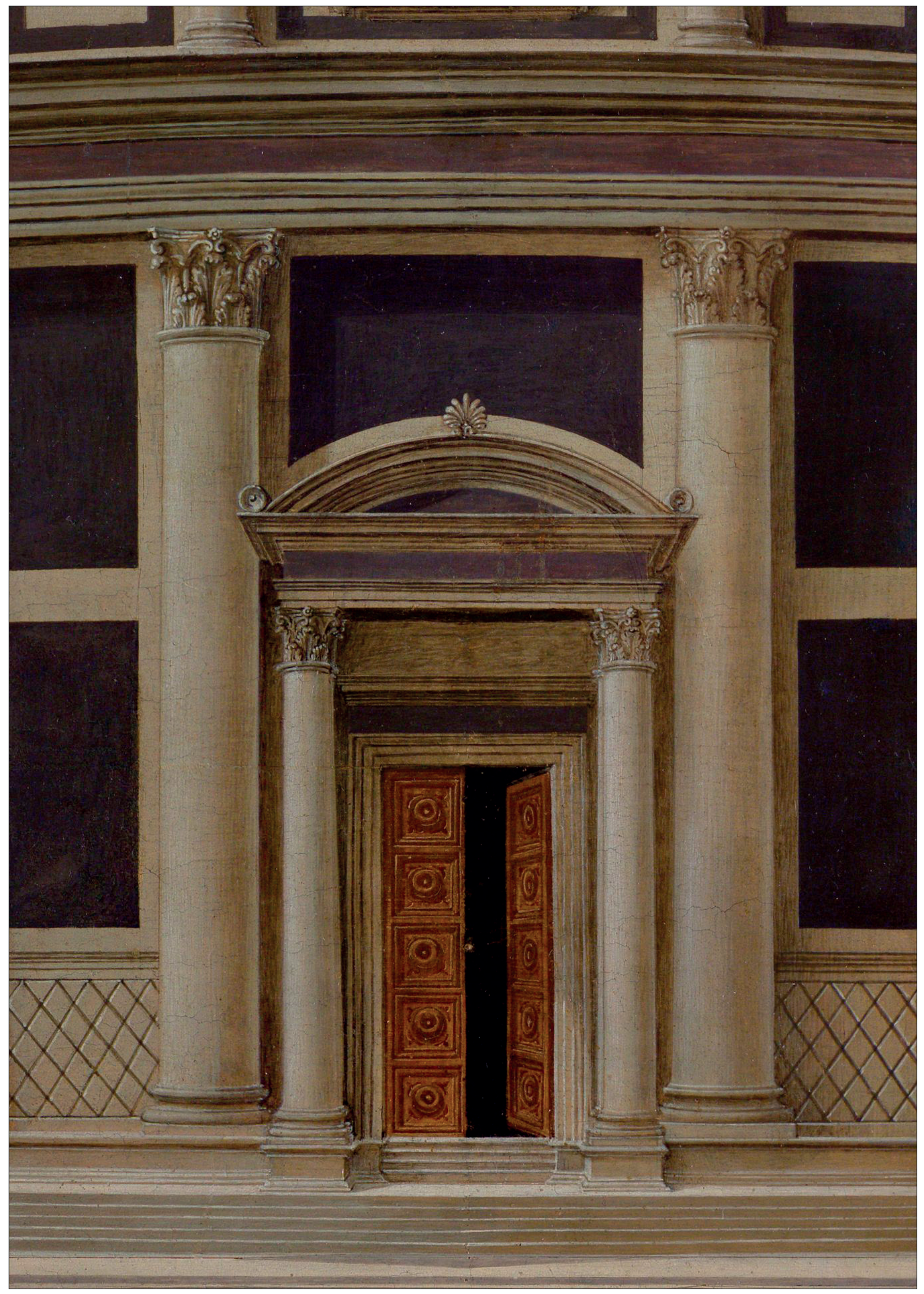




\title{
DUE QUAESTIONES VITRUVIANE RICONOSCIUTE: LA BASE ATTICA E IL CAPITELLO COMPOSITO NEL TERZO LIBRO DEL DE PROSPECTIVA PINGENDI DI PIERO DELLA FRANCESCA E UN PLAGIO CONCLAMATO DI LUCA PACIOLI
}

\author{
TWO RECOGNIZED VITRUVIAN PROBLEMS: \\ THE ATTIC BASE AND THE COMPOSITE CAPITAL \\ IN THE THIRD BOOK OF DE PROSPECTIVA \\ PINGENDI BY PIERO DELLA FRANCESCA AND \\ AN EVIDENT PLAGIARISM BY LUCA PACIOLI
}

\author{
Francesco P. Di Teodoro ${ }^{1}$ \\ DOI: http://dx.doi.org/10.5944/etfvii.2019.26181
}

A James (Jim) Banker

«in hoc octogesimo aetatis suae calculo»

\begin{abstract}
Sommario
Il saggio prende in considerazione due problemi vitruviano-pierfrancescani rimasti sino a oggi senza spiegazione e neppure riconosciuti come tali dai moderni esegeti del De prospectiva pingendi: la lunghezza del plinto della base attica (Libro III, propositio sexta) e la dimensione della concavità dell'abaco del capitello composito (Libro Ill, propositio septima).

In ambedue i casi i dati numerici danno conto dell'obbligata impossibilità di Piero nell'offrire soluzioni ai passi guasti dei codici vitruviani, tutti con numerosi errori. La condizione di Piero è la stessa di Leon Battista Alberti: nessuno dei due è un filologo. Lo studio rende ragione anche delle oscillazioni dimensionali proposte da Alberti per il lato del plinto.

Il saggio è occasione per «smascherare» un plagio di Luca Pacioli proprio in relazione ai due temi principali dell'intervento.
\end{abstract}

1. Politecnico di Torino (Professore ordinario di Storia dell'architettura) e Museo Galileo, Firenze. C. e.: francescopaolo.diteodoro@tin.it

Il testo è rielaborazione di un intervento presentato al convegno «Piero della Francesca and disegno», London, The Courtland Institute of Art, 19-20 June 2015, organised by J.R. Banker, T. Henry, M. Brüggen Israels, S. Nethersole, N. Silver, C. Campbell. Sono grato a Tom Henry per avermi consentito di pubblicarlo. Ringrazio l'amico Howard Burns che ha discusso con me l'argomento di questo saggio e, come di consueto, è stato generoso nei suggerimenti. 


\section{Parole chiave}

Piero della Francesca; Vitruvio; Proiezioni ortogonali; De re aedificatoria; De architectura; De prospectiva pingendi; Base attica; Capitello composito.

\section{Abstract}

The essay takes into consideration two problems related to Vitruvius and Piero della Francesca that have remained unexplained up to now and are not even recognized as such by the modern exegetes of De prospectiva pingendi: the length of the plinth of the Attic base (Book III, propositio sexta) and the size of the concavity of the abacus of the composite capital (Book III, propositio septima).

In both cases the numerical data reveal Piero's impossibility to offer solutions to faulty passages of the Vitruvian codices, all with numerous errors. Piero's condition is the same as Leon Battista Alberti: neither is a philologist. The study also explains the dimensional oscillations proposed by Alberti for the plinth side.

The essay is an opportunity to «unmask» a plagiarism by Luca Pacioli precisely in relation to the two main themes examined here.

\section{Keywords}

Piero della Francesca; Vitruvius; Orthogonal projections; De re aedificatoria; De architectura; De prospectiva pingendi; Attic base; Composite capital. 
UNO DEGLI ELEMENTI di maggior fascino nei dipinti a sfondo architettonico di Piero della Francesca è costituito dalla profusione di marmi e commessi sontuosi di geometrico rigore: porfido verde, porfido rosso, pavonazzetto, alabastro cotognino, portasanta, giallo antico, rari alabastri.

Tale ricchezza di ornamenti non è soltanto un riferimento all'arte e all'architettura classiche dovuto a quanto l'artista di Borgo Sansepolcro aveva potuto vedere e studiare nel corso dei suoi due soggiorni a Roma ${ }^{2}$, o un'allusione allo splendore dei rivestimenti parietali del vestibolo e dell'interno di Hagia Sophia - struggentemente celebrati e rimpianti dagli esuli di Bisanzio anelanti al vóбтоৎ - o memoria della sua esperienza fiorentina della fine degli anni Trenta del secolo: essa riflette anche la conoscenza del De architectura di Vitruvio.

Infatti, se già Leonardo Bruni nella Laudatio florentine urbis (ca I403-I4O4) aveva esaltato la città toscana come caratterizzata da «marmorea templa» ${ }^{3}$ e se il Battistero di San Giovanni, totalmente rivestito di marmo all'esterno come all'interno, altro non era, secondo la storiografia fiorentina, se non un tempio dedicato a Marte (Villani, Il, 5, IO-3I) - identificazione riaffermata dal Bruni nell'Historia florentini populi (circa I4I5) ${ }^{4}$ - dagli esempi della Città del Fiore derivava la facciata del tempio di Venere nel Ritrovamento e prova della vera Croce $^{5}$, al centro della parete sinistra del coro di San Francesco ad Arezzo; ma certamente il trattato di Vitruvio giustificava, con un puntuale riferimento radicato nella tradizione artistico-costruttiva romana, il ricorso al marmo, il solo materiale capace di conferire prestigio ai templi attraverso la magnificenza e il dispendio di mezzi:

[...] nam q(uat)tuor locis sunt ædiu(m) sacrar(um) marmoreis operibus ornate dispositiones, e quibus $p(r o)$ prie de is no(m)i(n)atio(n)es clarissima fama no(m)i(n)antur $[\ldots]$ id vero si marmoreu(m) fuisset, ut $h(a b e)$ ret que $(m) a d m o d u(m)$ ab arte subtilitatem sic a magnifice(n)tia \& impe(n)sis auc(torita)tem, i(n) primis \& summis operibus no(m)i(n)aret(ur) ${ }^{6}$.

Il rinvio a Vitruvio non stupisce, d'altro canto, giacché dopo Euclide e Archimede, l'antico architetto è l'auctor più citato da Piero, direttamente o indirettamente. Infatti, dalla prefazione al Terzo Libro del De architectura ${ }^{7}$, Piero trae sia le citazioni

2. Si pensi soprattutto all'interno del Pantheon e alle grandi rotae di San Lorenzo fuori le Mura e di San Giovanni in Laterano.

3. Cfr. Di TeOdoro, Francesco Paolo, «"Marmorea templa": Firenze, identità romana e tutela identitaria», in Burns, Howard e Mussolin, Mauro (eds.), con la collaborazione di Altavista, Clara: Architettura e identità locali, II. Firenze, Olschki, 2013, 449-469.

4. La selettività del marmo per le chiese è conservazione e specchio dell'identità romana di Firenze.

5. Segnalo, solo di tangenza, come il motivo delle sfere entro tondi dipinto da Piero abbia consonanza con le patene campite su un fondo che imita marmi venati, nella porzione basamentale degli affreschi di Paolo Uccello e Scuola nel chiostro di San Miniato, oltre che precisi riferimenti nell'architettura tardogotica; ad esempio nella facciata della Ca' d'Oro a Venezia.

6. Vitr. VII, praef., 16-18. Cito dall'editio princeps (Firenze, BNC, C.3.25). Vitruvio, d'altro canto, è in linea con gli auctores quali Virgilio, Servio, Ovidio, Properzio che avevano affermato che i templa erano di marmo.

7. Cfr. Vitr. III, praef., 2. Cito dall'editio princeps (Firenze, BNC, C.3.25): «Maxime aut(em) id a(n)i(m)advertere possumus ad antiquis statuariis \& pictoribus, q(uod) ex his qui dignitatis notas et co(m)mendatio(n)is gra(tia)m habueru(n)t, aeterna memoria ad posteritate(m) su(n)t permane(n)tes, uti Myron Polycletus, Phidias, Lysippus, ceteriq(ue) qui nobilitate(m) ex arte sunt consecuti. Namq(ue) ut in civitatibus magnis aut regibus aut nobilibus civibus opera fecerunt, ita id sunt adepti. At qui non minore studio \& ingenio sollertiaq(ue) fueru(n)t nobilibus, \& 
nell'introduzione al Terzo Libro del De prospectiva pingendi ${ }^{8}$ sia quelle presenti nella dedicatoria a Guidubaldo da Montefeltro nel tardo Libellus de quinque corporibus regularibus (Cod. Vat. Urb. Lat. 632) ${ }^{9}$ composto, come lo stesso artista-matematico scrive, «in hoc ultimo aetatis meae calculo». In questo secondo caso - lo ha riconosciuto per prima Margaret Daly Davies - si tratta addirittura di una parafrasi ${ }^{10}$.

Come noto il foglio con la dedicatoria venne aggiunto in un secondo tempo al codice vaticano, il che starebbe a suggerire l'esistenza di una precedente lettera, sostituita (in fretta?) dopo la scomparsa di Federico da Montefeltro. Ė verosimile, però, che il testo eliminato non sia stato del tutto rimpiazzato da uno nuovo, ma solo modificato e, per dir così, aggiornato. Infatti, la parafrasi vitruviana suggerirebbe una dedica proprio all'indirizzo di Federico che, stanti le sue non comuni competenze architettoniche, avrebbe immediatamente riconosciuto il celebre incipit vitruviano gustando, perciò, l'anticus sapor della lettera.

Infine, con il ricorso a Vitruvio, così nel Libellus come già nel De prospectiva pingendi, Piero continuava ad accreditarsi come architetto.

\section{LA BASE ATTICA}

Nell'architettura dipinta Piero raffigura sempre colonne o paraste con la base attica, soggetto, quest'ultimo, che l'artista toscano tratta anche nella propositio sexta del Terzo Libro del De prospectiva pingendi, disegnandone in doppia proiezione ortogonale pianta e prospetto, grafici necessari per poter poi tracciare la veduta in prospettiva $^{\text {II }}$ (Figure I-4), secondo il metodo dell'intersegazione esposto, per l'appunto, nel Terzo Libro del trattato:

Per seguire l'ordine dato, fa' p(ri)ma la basa i(n) p(ro)pria forma, che se veggha solo una faccia, co(m)mo vederai nella figura dell'alteçça. La quale basa vole essere alta la metà della groseçça della colonna, et larga da piè quanto hè grossa la colonna, et più doi quinti, la quale largheça sia GH. Poi dividi l'alteçça in dodici parti equali, delle quali

humili fortuna civibus no(n) minus egregie perfecta fecerunt opera, nulla(m) memoriam sunt assecuti, q(uod) hi no(n) $a b$ industria neq(ue) artis solertia sed a felicitate fueru(n)t diserti, ut Hellas Atheniensis, Dhion Corynthius, Miagrius Phoceus, Pharax Ephesius; Boedas Byzantius, etiamq(ue) alii plures. No(n) minus ite $(\mathrm{m})$ pictores, uti Aristomenes Thasius, Polydes \& Andramites, Niteomagnius ceteriq(ue), quos neq(ue) industria neq(ue) artis studiu(m) neq(ue) solertia defecit $[\ldots] »$.

8. «La quale [prospettiva] seguitando molti antichi dipinctori acquistaro p(er)petua laude; co(m)mo Aristomenes Thasius, Polides, Apello, Andramides, Nitheo et molti altri» (De p.p., III, proemio: Mss. Regg. A 41/2, c. 38v).

9. Città del Vticano, Biblioteca Apostolica Vaticana, Cod. Vat. Lat. 632, c. Ir: «Inter antiquos pictores et statuarios, GVIDO princeps insignis, Policretum, Phidiam, Mironem, Praxitelem, Apellem, Lisippum ceterosq(ue) qui nobilitatem ex arte sunt consecuti, non ob aliud digniores fuisse, \& apud suos maiorem gratiam, apud v(er)o posteritatem memoriam \& famam diuturniorem, Aristomene Thasio, Polide, Chione, Pharaxe, Boeda ceterisq(ue), qui non minori artis studio, ingenio, solertia \& industria fuerunt, habuisse p(er)hibent, nisi q(uo)d ii aut civitatibus magnis, aut regibus, aut principibus virtutis experimenta opera fecerunt».

10. Cfr. Daly Davis, Margaret: Piero della Francesca's Mathematical Treatises, The «Trattato d'abaco» and «Libellus de quinque corporibus regularibus». Ravenna, Longo, 1977, 44-45.

11. Per la costruzione della base seguendo il dettato pierfrancescano si veda CAMEROTA, Filippo, «Scheda VI.1», in Camerota, Filippo, Di Teodoro, Francesco Paolo e Grasselli, Luigi (eds.): Piero della Francesca, il disegno tra arte e scienza, cat. della mostra (Reggio Emilia 14 marzo-13 giugno 2015). Milano, Skira, 2015, 356-357. 


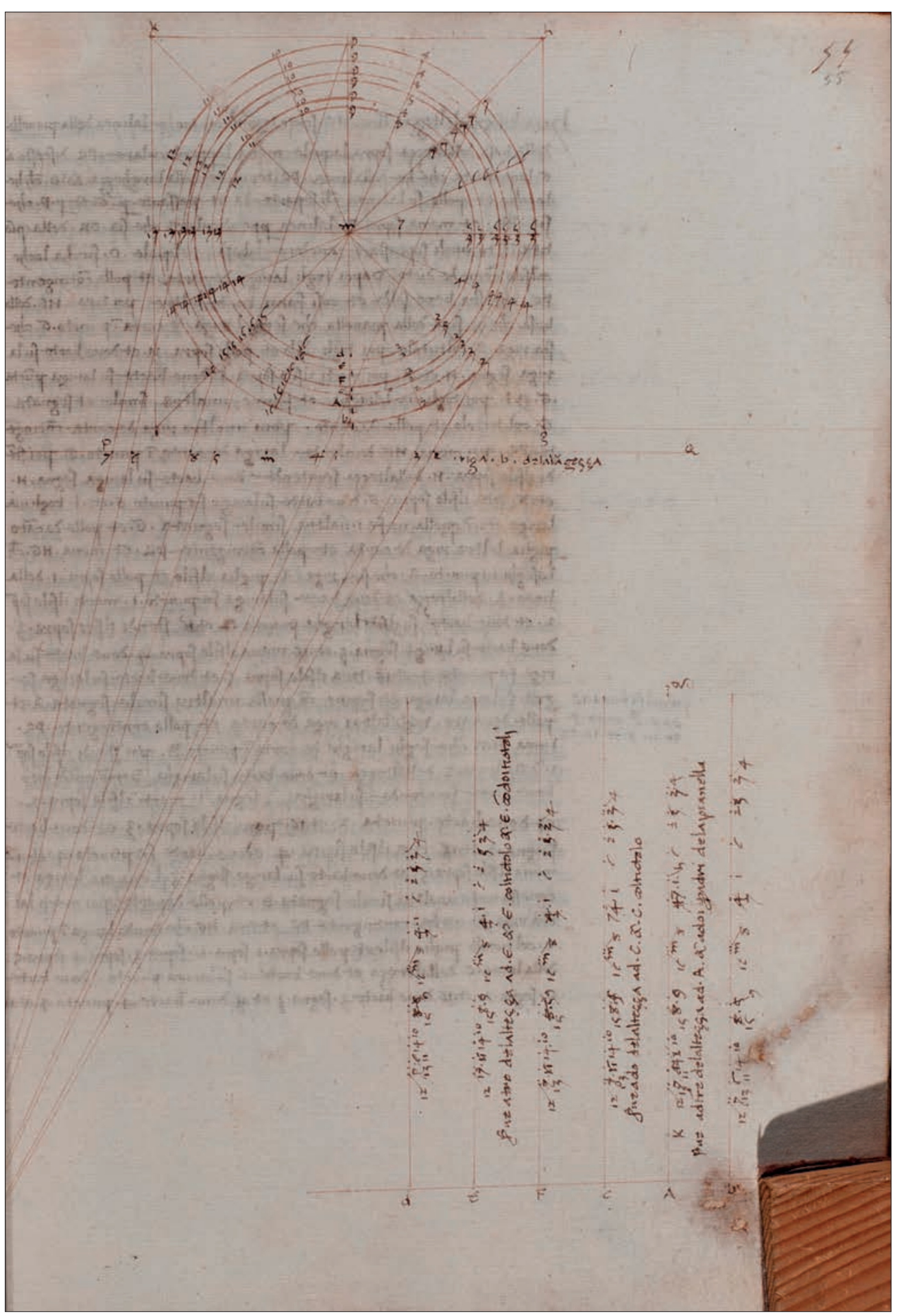

FIGURA 1. PIANTA DI UNA BASE ATTICA. PIERO DELLA FRANCESCA, DE PROSPECTIVA PINGENDI, REGGIO EMILIA, BIBLIOTECA COMUNALE «A. PANIZZI», COD. REGG. A 41/2, C. 55R. 
pon(n)e quattro sopra $G$, che sia $A$, p(er) la pianellecta che hè dicta plintho. Et tre parti ne poni sopra $A$, che sia $C p(e r)$ lo tondo, ch(e) hè dicto thoro; et meçça de una de queste parti poni sopra $C$, che sirà pur $C$. Et doi parti ne poni sopra $C$, che sia $E$ p(er) la cinta; et meçça parte sopra $E, p(e r)$ lo regolecto che hè sopra la cinta che sia $E$; et il resto hè $\mathrm{p}(\mathrm{er})$ lo tondino de sopra, che sia pure $\mathrm{E}^{12}$.

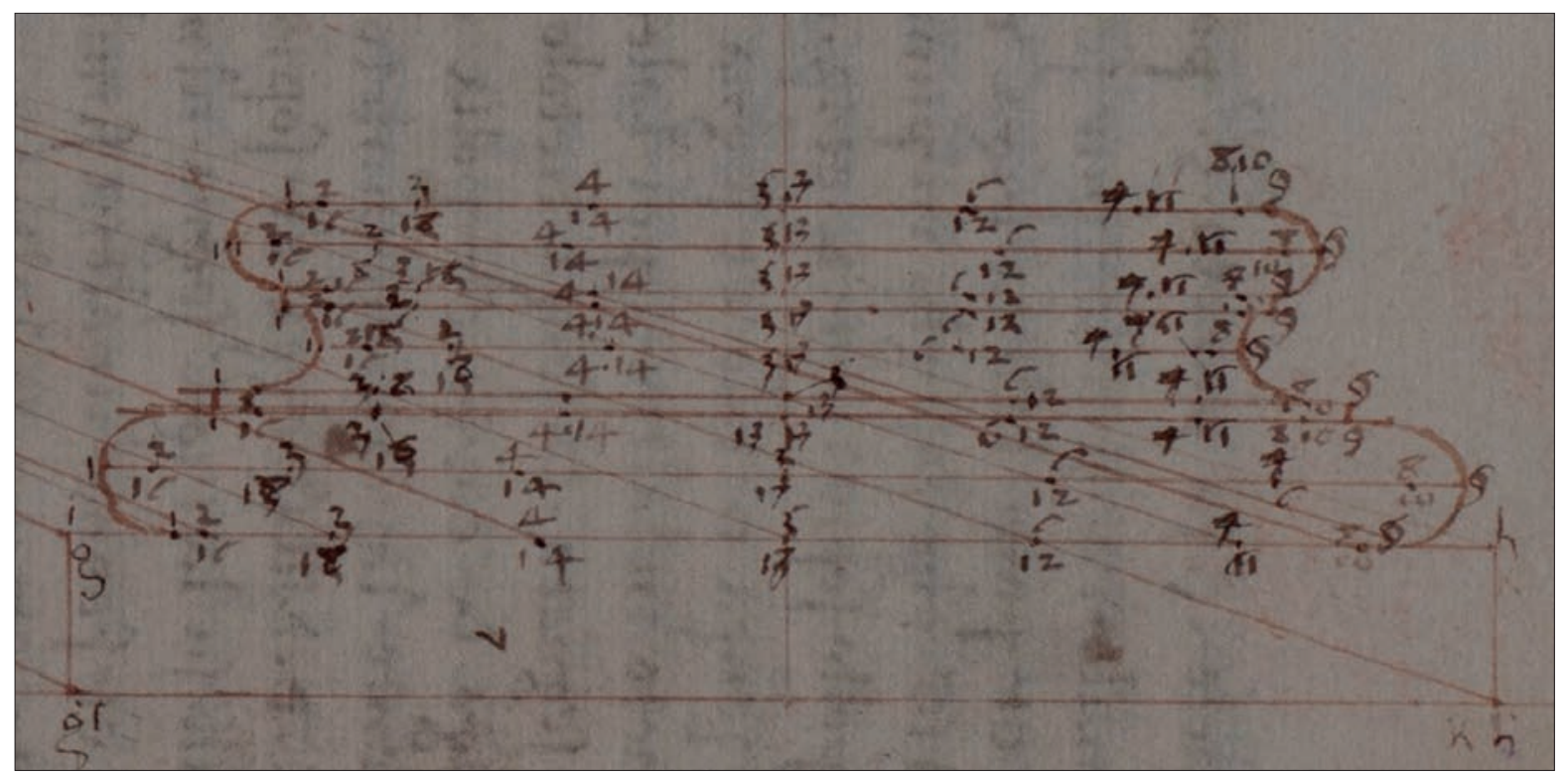

FIGURA 2. PROSPETTO DI UNA BASE ATTICA. PIERO DELLA FRANCESCA, DE PROSPECTIVA PINGENDI, REGGIO EMILIA, BIBLIOTECA COMUNALE «A. PANIZZI», COD. REGG. A 41/2, C. 56V, PARTICOLARE.

Non sorprende, ed è già stato notato, che le proporzioni della base siano desunte da un codice del De architectura di Vitruvio, testo imprescindibile per chiunque si fosse voluto occupare di architettura:

\begin{abstract}
His perfectis in suis locis spire collocent(ur) heaeq(ue) ad symmetriam sic perficiant(ur) uti crassitudo cum plinthio sit Columne ex dimidia crassitudine proiecturamq(ue), quam graeci esphoran vocant, habea(n)t sexta(n)tem; ita $\mathrm{t}(\mathrm{a}) \mathrm{n}(\mathrm{tum})$ lata \& longa erit columne crassitudinis unius \& dimidie. Altitudo eius si attigurges erit, ita dividatur ut superior pars tertia parte sit crassitudinis columne, reliquum plintho relinquat(ur). Dempta plintho reliquu(m) dividat(ur) in $\mathrm{p}(\mathrm{ar}) \mathrm{tes}$ quatuor fiatq(ue) sup(er)ior chorus [torus quartae, reliquae tres aequaliter dividantur et una sit inferior torus] altera pars $\mathrm{cu}(\mathrm{m})$ suis quadris scotica quam greci trochilion dicunt. ${ }^{13}$
\end{abstract}

12. Cito da: Reggio Emilia, Biblioteca Comunale «A. Panizzi», Mss. Regg. A 41/2, c. 53r. E cfr. PIero della Francesca: De prospectiva pingendi, Gizzı, Chiara (ed.). Venezia, Edizioni Ca' Foscari, 2016, 228. Per la datazione della traduzione latina del trattato prospettico di Piero si veda ora BERTOLINI, Lucia: «Appunti su cronologia e circolazione del De prospectiva pingendi》, in CAMEROTA, Filippo e DI TEODORO, Francesco Paolo (eds.): Piero della Francesca, la seduzione della prospettiva, catalogo della mostra (Sansepolcro, 21 marzo 2018-6 gennaio 2019). Venezia, Marsilio, 2018, 25-31.

13. Cito dall'editio princeps (Firenze, BNC, C.3.25) integrata, quanto a una omissione, da un passo del Cod. Magl. XVII.5, c. 19r, della stessa biblioteca, un ms. datato 1453. 


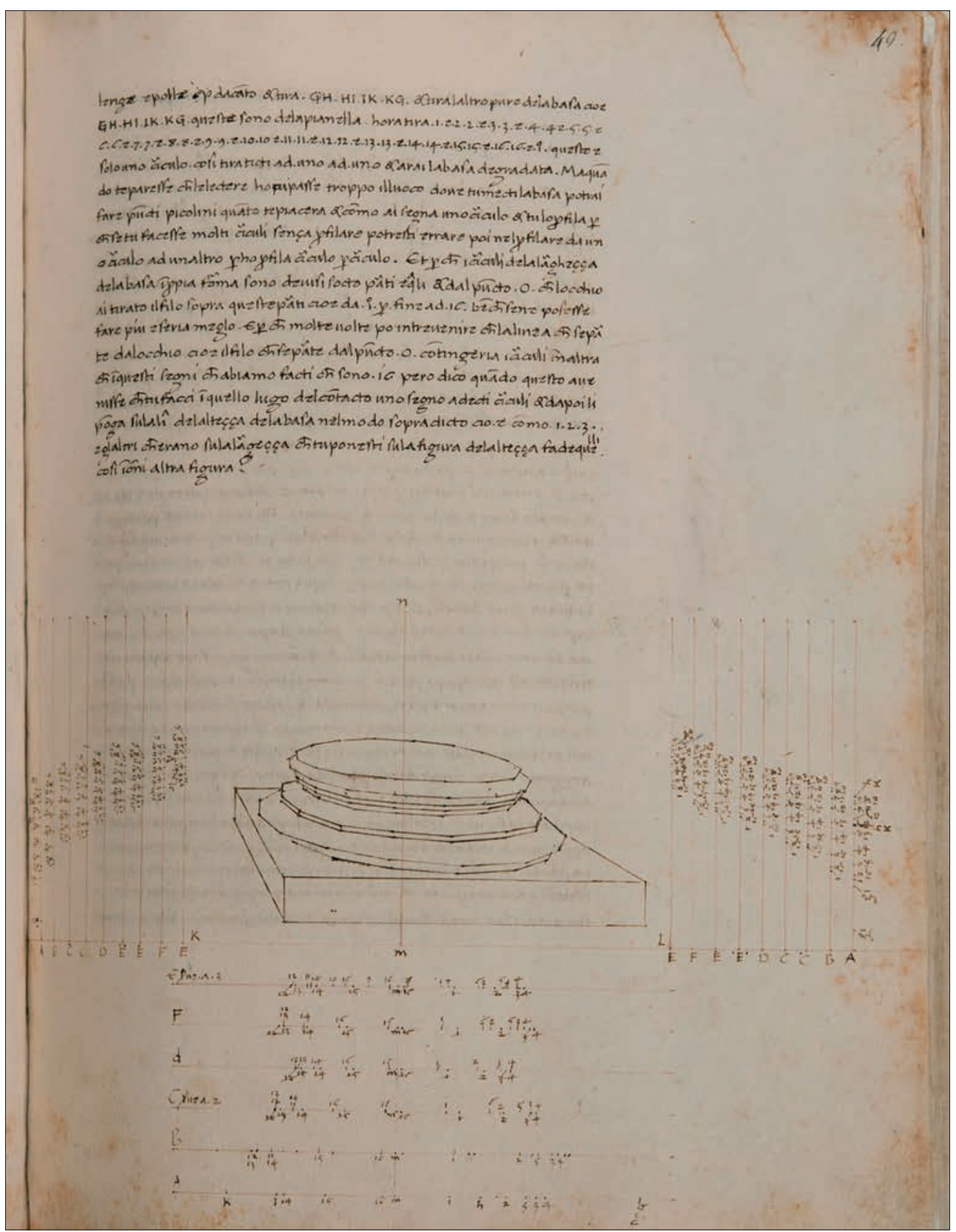

FIGURA 3. PROSPETTIVA DI UNA BASE ATTICA. PIERO DELLA FRANCESCA, DE PROSPECTIVA PINGENDI, PARMA, BIBLIOTECA PALATINA, MS. PARMENSE 1576, C. 49 R.

La base di Piero, dunque, ha l'altezza indicata da Vitruvio (III, 5, I-2) e ripetuta da Leon Battista Alberti $(\mathrm{VII}, 7)^{\mathrm{I}}$, cioè pari al raggio della colonna all'imoscapo, misurato sopra l'apofige e non alla cintura (Figura 5).

14. La base dorica albertiana, come noto, segue le proporzioni della base attica vitruviana: cfr. L.B. ALBERTI, De re aed., VII, 7. 

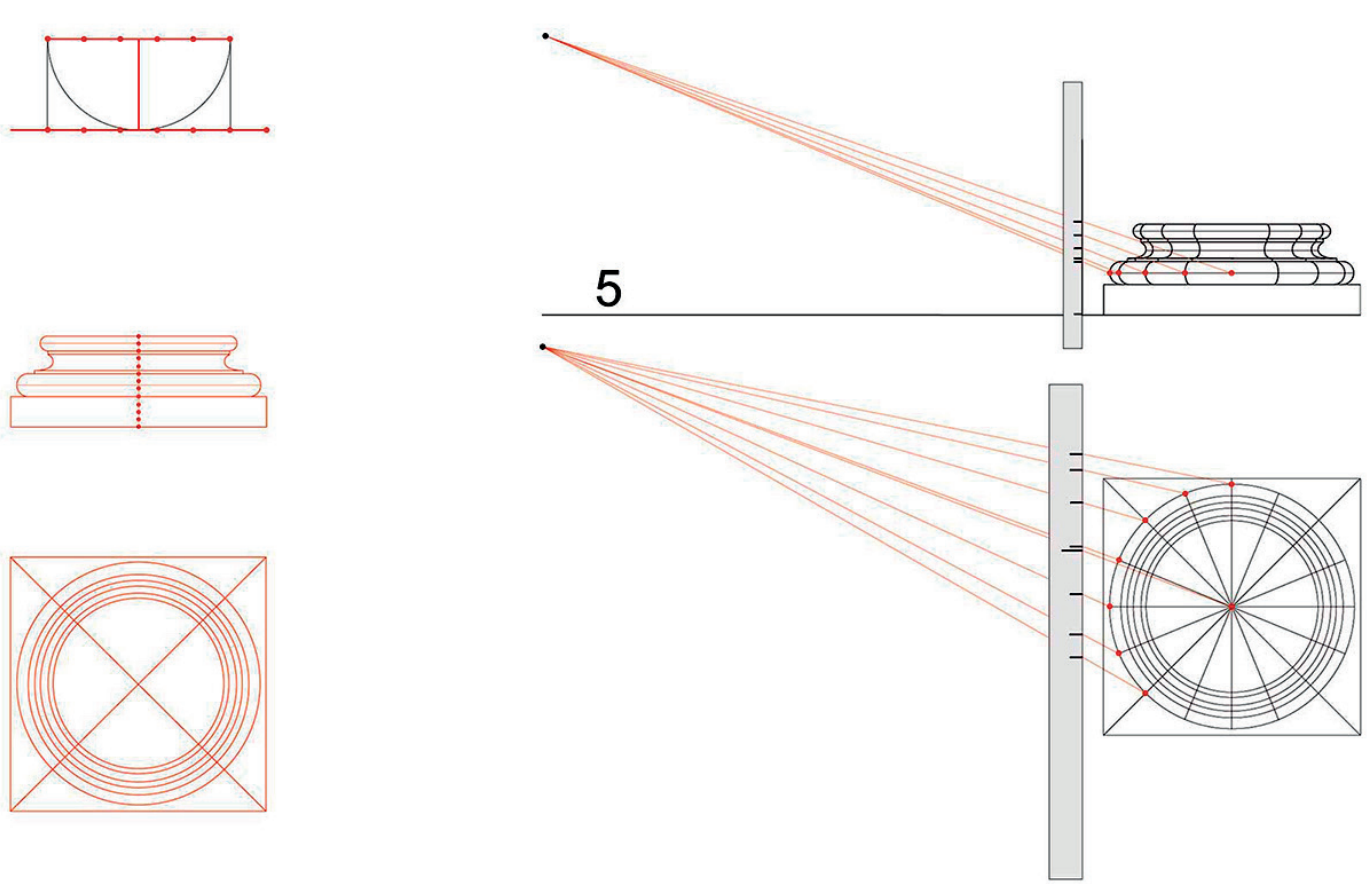

2

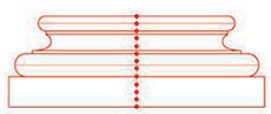

3

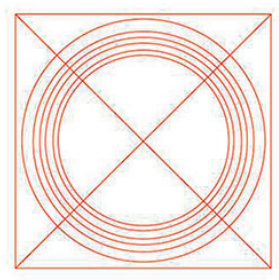

6

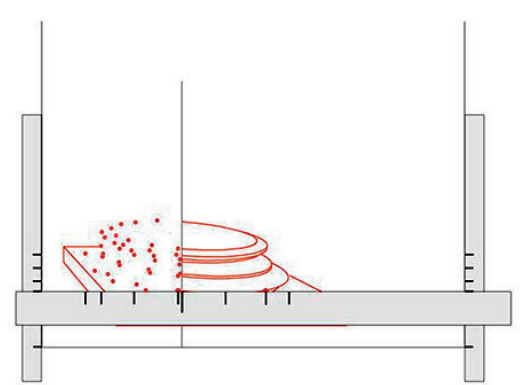

FIGURA 4. COSTRUZIONE PROSPETTICA DELLA BASE ATTICA DA DE PROSPECTIVA PINGENDI (F. CAMEROTA). DA PIERO DELLA FRANCESCA, IL DISEGNO TRA ARTE E SCIENZA...MILANO 2015, P. 222.

Anche le altezze del plinto e dei due tori, pari rispettivamente a I/6, I/8 e I/I2 del diametro all'imoscapo, sono le medesime di Vitruvio e di Alberti.

Se Vitruvio non dà le dimensioni dei listelli che precedono e seguono la scozia, ma una misura complessiva - $\mathrm{I} / 8$ del diametro -, Alberti le indica ciascuna pari a I/7 del totale mentre Piero le dice pari a I/6.

Anche l'altezza del plinto è la stessa data da Vitruvio e da Alberti: I/6 del diametro della colonna all'imoscapo. Piero ne specifica, però, la lunghezza del lato pari a 7/5 del diametro, cioè I,4 d; egli, infatti, scrive che il lato è largo «da piè qua(n)to è grossa la colon(n)a, più doi qui(n)ti le la grosseçça de la colo(n)na» (Cod. Parm. 1576, c. 44r), dove «doi quinti» è la dimensione complessiva della sporgenza del plinto rispetto al diametro della colonna all'imoscapo (dunque $\mathrm{I} / 5$ da ambedue le parti):

$$
L_{\text {Piero }}=\mathrm{d}+\mathrm{I} / 5 \mathrm{~d}+\mathrm{I} / 5 \mathrm{~d}=7 / 5 \mathrm{~d}=\mathrm{I}, 4 \mathrm{~d}
$$




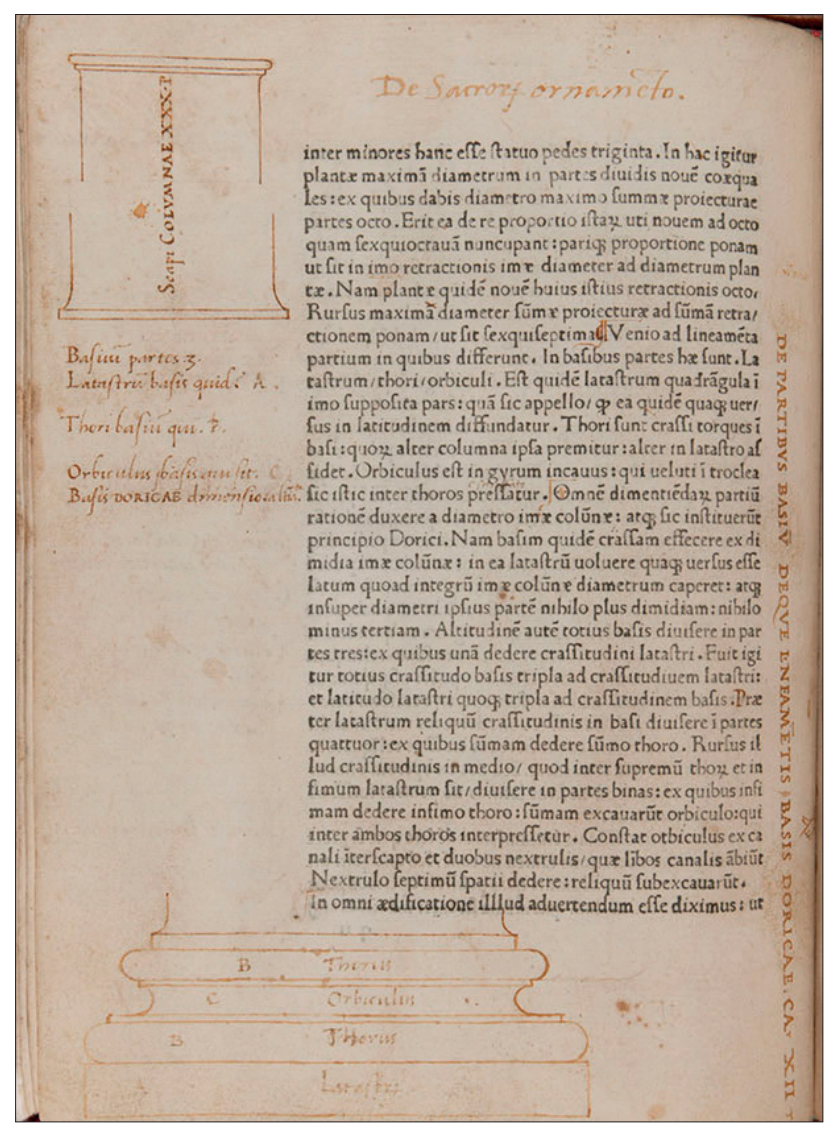

FIGURA 5. PROSPETTO DI UNA BASE ATTICA. IN L.B. ALBERTI, DE RE AEDIFICATORIA, FIRENZE 1485, LIBRO VII, C. 118V. TORINO, ARCHIVIO ARCIVESCIVILE, S. C.

Nel quinto capitolo del Terzo Libro del De architectura (Vitr. III, 5, I-2) Vitruvio scrive, invece, che il lato del plinto è $3 / 2$ del diametro, cioè una volta e mezzo il diametro (I,5 d), dove, dunque, l'ह̉кфорá (esphoran nella princeps) è complessivamente $1 / 2$ del diametro, cioè a dire $1 / 4 \mathrm{e}^{1 / 4}$ da ogni banda rispetto all'asse:

$$
\mathrm{L}_{\text {Vitruvio }}=\mathrm{d}+1 / 2 \mathrm{~d}=\mathrm{d}+1 / 4 \mathrm{~d}+1 / 4 \mathrm{~d}=3 / 2 \mathrm{~d}=\mathrm{I}, 5 \mathrm{~d}
$$

mentre l'Alberti afferma che esso è compreso tra una volta e un terzo (I,33 d) e una volta e mezzo il diametro ( $\mathrm{I}, 5 \mathrm{~d}$ ): «in ea latastr(um) voluere quaq(ue) versus esse latum, quoad integru(m) imae colu(m)nae diametrum caperet atq(ue) insuper diametri ipsius parte $(\mathrm{m})$ nihilo plus dimidiam nihilo minus tertiam $»^{15}$ :

15. Cito da Alberti, Leon Battista: De re aedificatoria. Firenze, Niccolò di Lorenzo Alamanni, 1485 (esemplare dell'Archivio arcivescovile di Torino, s. collocazione, c. 118v). Per tale esemplare con un apparato notevole di disegni a penna cfr. DI TEOdoro, Francesco Paolo: «Intorno a una princeps del De re aedificatoria», in CALzonA, Arturo et alii: Leon Battista Alberti, architetture e committenti, atti dei convegni internazionali. Firenze, Rimini, Mantova, 12-16 ottobre 2004), II. Firenze, Olschki, 2009, 589-615. 


$$
\begin{aligned}
& \mathrm{d}+\mathrm{I} / 3 \mathrm{~d} \leq \mathrm{L}_{\text {Alberti }} \leq \mathrm{d}+1 / 2 \mathrm{~d} \rightarrow \mathrm{d}+\mathrm{I} / 6 \mathrm{~d}+\mathrm{I} / 6 \mathrm{~d} \leq \mathrm{L}_{\text {Alberti }} \leq \mathrm{d}+1 / 4 \mathrm{~d}+1 / 4 \mathrm{~d} \rightarrow \mathrm{I}, 33 \mathrm{~d} \leq \\
& \mathrm{L}_{\text {Alberti }} \leq \mathrm{I}, 5 \mathrm{~d}
\end{aligned}
$$

Le discrepanze maggiori fra Piero, Vitruvio e Alberti si riscontrano, dunque, nella lunghezza del lato del plinto, o meglio, nella dimensione dell'żкфорá. Quale ne è la ragione? E perché Alberti non dà la stessa grandezza di Vitruvio (I,5 d), ma suggerisce un'oscillazione che, peraltro, giustifica il valore dato da Piero (che è compreso tra $\mathrm{I}, 33 \mathrm{~d}$ e $\mathrm{I}, 5 \mathrm{~d})$ ?.

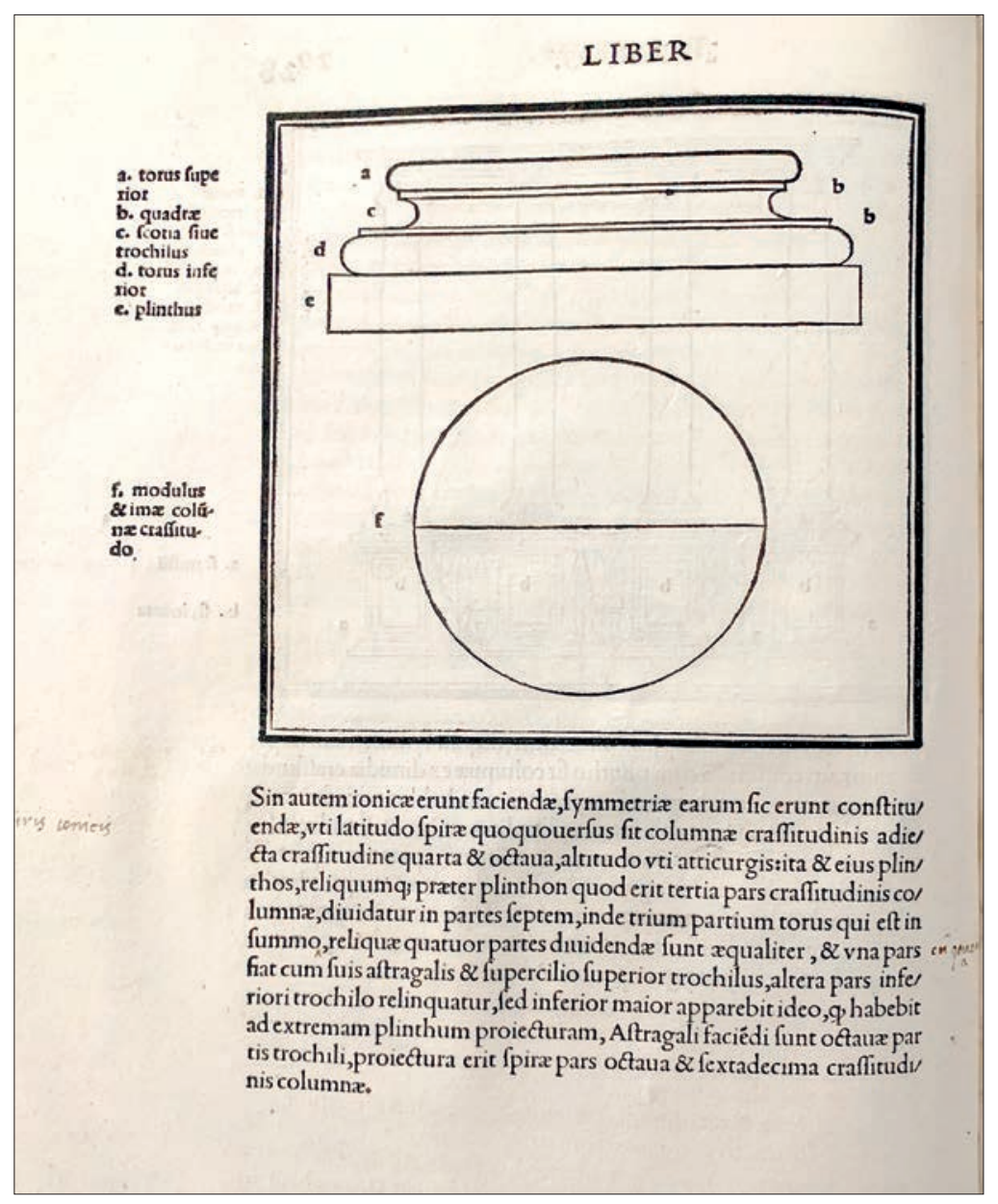

FIGURA 6. PIANTA E PROSPETTO DI UNA BASE ATTICA. IN M. VITRUVIUS PER IOCUNDUM ..., VENEZIA 1511, C. $28 \mathrm{~V}$.

Se ce ne stiamo a quanto si legge nelle moderne edizioni critiche e nelle attuali traduzioni - cioè ai testi ai quali sempre, senza eccezione, sono soliti riferirsi gli storici dell'architettura e gli storici dell'arte - siamo destinati a non trovare risposte e a formulare ipotesi più o meno suggestive. E, anzi: il problema neppure esiste.

L'architettura studiata filologicamente - una modalità su cui gli studiosi convergono - imporrebbe un analogo atteggiamento filologico nei confronti delle fonti scritte (in questo caso Vitruvio); è un paradosso, ma nessuno lo ha mai praticato, tutt'altro. L'errore più grave che si possa compiere è ricorrere, come tuttora si fa, alle edizioni critiche del De architectura e alla loro luce giudicare l'architettura del XV e del XVI 
secolo, conferendo o meno patenti di correttezza vitruviana ora all'uno ora all'altro artefice. Ebbene, anche per Vitruvio è del tutto fuorviante il ricorso persino alla migliore delle edizioni critiche per la semplice ragione che quel testo non è mai stato noto nel corso dei secoli così come noi oggi abbiamo il privilegio di conoscerlo. Disporre del testo emendato degli errori di trasmissione dei testimoni pone il moderno lettore di fronte all'antico trattato come se si trovasse allo scrittoio stesso di Vitruvio. In tal modo, però, si oscura il tempo e si cancella la storia poiché il De architectura è stato letto nei secoli solo ed esclusivamente attraverso la lente di codici guasti e lacunosi: ma sono proprio gli errori ad aver condizionato e orientato la storia architettonica occidentale. Ogni testimone, grazie al nome quasi mitico del suo autore, ha costituito il riferimento principe per coloro che quel manoscritto hanno posseduto o avuto l'occasione di leggere, copiare o consultare. Dunque: quot homines tot sententiae: tanti codici, tanti Vitruvio. Ogni esemplare, per guasto che fosse, era la verità e dettava le normae.

La ragione, dunque, dei valori diversi tra Piero, Alberti e Vitruvio relativamente alla lunghezza del lato del plinto della base attica risiede nel passo contraddittorio presente nella tradizione manoscritta e nelle edizioni quattrocentesche del De architectura (ma non nelle edizioni critiche moderne: è la ragione per cui il problema non è mai stato riconosciuto, né affrontato, ma oscurato alla luce abbagliante di un testo emendato e senza errori: una sorta di copertura antibiotica che nasconde la natura di un male alle indagini cliniche). Vitriuvio scrive (cito dall'editio princeps, ma è lo stesso nei codici):

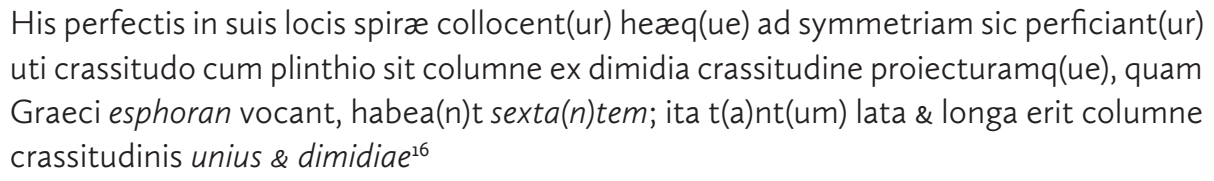

«Sextantem» vuol dire I/6, quindi, per l'intera lunghezza I/6 più I/6 (la misura va sommata due volte in relazione all'asse della colonna), cioè $\mathrm{I} / 3$, da cui la lunghezza del plinto pari a I+I/3, ossia I, 33 volte il diametro (esattamente la dimensione inferiore data da Alberti). In tal modo, però, non c’è coincidenza tra «sextantem» e «dimidiae». Perché il lato del plinto sia «unius \& dimidiae» occorrerebbe correggere sextantem con quadrantem (è quello che fa, ad esempio, Fra Giocondo nella sua edizione illustrata del I5II) ${ }^{17}$ (Figura 6). In tal caso quadrantem, che vuol dire $1 / 4$, darebbe come lunghezza I+ $1 / 4+1 / 4$ cioè 1,5 volte il diametro (esattamente il limite superiore suggerito da Alberti, ma anche da Vitruvio alla fine del passo in esame).

Si capisce, intanto, la doppia possibilità offerta da Alberti che, non essendo un filologo, non abituato a collazionare i testi e neppure a fare congetture, di fronte al dilemma taglia la testa al toro e accoglie entrambe le possibilità: mantiene la

16. Miei i corsivi.

17. Cfr. Gıocondo, Giovanni (ed.): M. Vitruvius ... solito castigatior factus cum figuris. Venezia, loannis de Tridino alias Tacuino, 1511, c. 28r: "His perfectis in suis locis spirae collocentur, eaeq(ue) ad symmetriam sic perficiantur, uti crassitudo cum plintho sit columnae ex dimidia crassitudine, proiecturamq(ue) quam graeci દ̉k $\phi \circ \rho a v$ vocitant, habeant quadrantem. Ita tum lata \& longa erit columnae crassitudinis unius \& dimidiae». 
lunghezza del plinto entro due limiti, l'uno che vede aggiunto al diametro della colonna all'imoscapo un sextantem l'altro che vi vede aggiunto un dimidium. Piero neppure lui un filologo -, per parte sua, cerca di tenere il lato del suo plinto all'interno del margine di variabilità di Alberti - di cui solo adesso sono chiare le motivazioni, mai fino a ora spiegate - e indica il valore di I,4. Tale grandezza, peraltro, è molto prossima a quella della lunghezza del plinto ionico (o efesio), pari a I,375 (lo stesso è in Alberti), di cui Vitruvio (Vitr., III, 3) tratta nel passo successivo a quello appena rammentato («Proiectura erit sperae pars octava \& sextadecima p(ar)s grassitudinis columne») $)^{18}$.

Può darsi che Piero si sia servito di un codice vitruviano guasto in cui la proiectura non era I/6 né $1 / 4$ ma I/5; tuttavia gli esemplari che ho esaminato sino a ora sono concordi nel tramandare sextantem. In ogni caso la soluzione del passo del De architectura, decisamente problematico e, come si è visto, contraddittorio, è stata data solo nel I9I2 da Fritz Krohn che ha suggerito di separare la lettera «S» dal resto nella parola sextantem ottenendo, quindi, $s$ (emissem) extantem, cioè «sporgente $\mathrm{di} 1 / 2$ », dato, dunque, in perfetta sintonia con «unius et dimidiae» ${ }^{\text {19 }}$.

\section{IL CAPITELLO COMPOSITO}

Nell'architettura dipinta Piero ricorre a più specie di capitelli, in particolare compositi: da quello all'antica con cornucopie in luogo di volute angolari della Resurrezione di Cristo ${ }^{20}$ (Figura 7) di Sansepolcro (impiegato anche da artisti suoi contemporanei) a quelli della Flagellazione e della Leggenda della vera croce.

Il capitello a otto volute della Flagellazione - che resta un unicum nella pittura pierfrancescana - è verosimilmente rielaborazione dei corinzi brunelleschiani o di quelli dipinti dall'Orcagna in Santa Croce ${ }^{21}$. João Basto ha segnalato non pochi esemplari della stessa foggia di quelli di Piero ${ }^{22}$.

18. Cito dall'editio princeps. Firenze, BNC, C.3.25

19. Cfr. Vitruvius: De architectura libri decem. KROHN, Fritz (ed.). Leipzig, Teubner, 1912 («Bibliotheca scriptorum Graecorum et Romanorum Teubneriana»).

20. Come noto il dipinto murale ci è giunto mutilo nella porzione inferiore, tanto che la scritta latina in caratteri capitali che l'accompagnava è decisamente frammentaria. Colgo l'occasione per proporne una integrazione pertinente al soggetto del dipinto. Rispetto alla proposta da me elaborata (in grassetto le lettere ancora comprensibili, benché incomplete): «PRO HVMANITATEM A MORTE RESVRREXIT»), ne ha proposta una pià congrua, nel rispetto dei margini, ai moduli delle lettere e della spaziatura, Giacomo Guazzini (al quale si deve anche la figura schematica e che ringrazio) che mi suggerisce: «PRO HVMANITATEM A MORTE RESVRGENS». II participio presente, piuttosto che il tempo al passato, illustra esattamente ciò che si vede: il Cristo risorto che sta uscendo dal sepolcro.

21. Da Andrea Orcagna - Tabernacolo in Orsanmichele - aveva attinto anche Masaccio per le piccole piramidi collocate nell'intradosso dell'arco introduttivo al sacello dipinto nella Trinità. Cfr. Di Teodoro, Francesco Paolo: «L'architettura della Trinità», in CAMEROTA, Filippo (ed.): Nel segno di Masaccio. L'invenzione della prospettiva, cat. della mostra (Firenze 16 ottobre 2001-20 gennaio 2002). Firenze, Giunti, 2001, pp. 47-51; Id., «'... che pare sia bucato quel muro': l'architettura «verosimile» della Trinità di Masaccio», in Cazzato, Vincenzo, Roberto, Sebastiano e Bevilacqua, Mario (eds.): La festa delle arti. Scritti in onore di Marcello Fagiolo per i cinquant'anni di studi, I. Roma, Gangemi, 2014, pp. 206-211.

22. BASTO, João: «The composite capitals in Piero della Francesca's Flagellation», in DALAI EMILIANI, Marisa e CURZI, Valter (eds.): Piero della Francesca tra arte e scienza, Atti del convegno internazionale di studi (Arezzo, 8-11 ottobre 1992, Sansepolcro, 12 ottobre 1992). Venezia, Marsilio, 1996, pp. 77-94. 


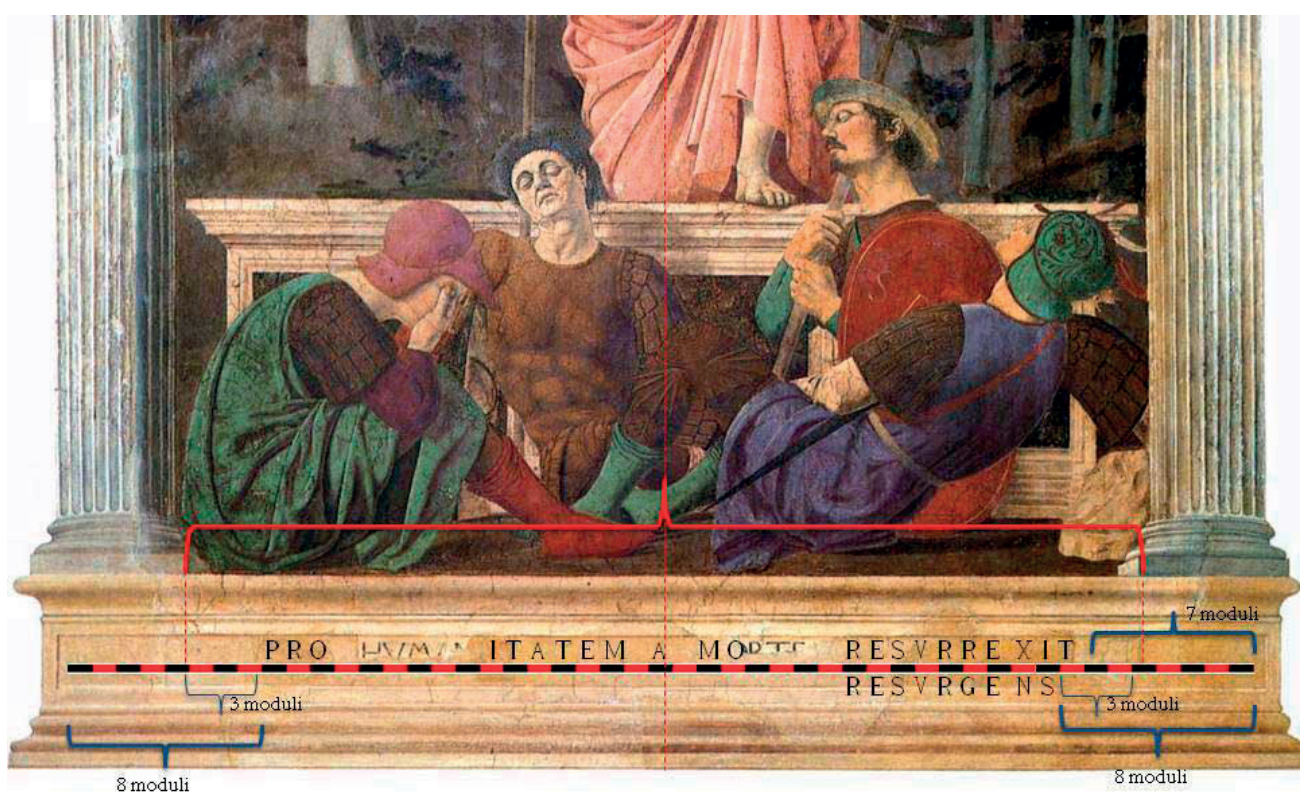

FIGURA 7. PROPOSTA DI LETTURA INTEGRATIVA ALLA BASE DELLA RESURREZIONE DEL MUSEO CIVICO DI SAN SEPOLCRO (F.P. DI TEODORO E G. GUAZZINI; DIS. DI G. GUAZZINI).

Di maggior momento, però, sono i capitelli compositi della Leggenda della vera croce, della cimasa del Polittico di Sant'Antonio e della Madonna di Williamstown.

Si tratta di un tipo all'antica noto da esemplari d'età imperiale e medievali, comuni nell'architettura romanica fiorentina (San Miniato, Battistero, Santissimi Apostoli) e nella pittura quattrocentesca (rammento soltanto la Madonna delle ombre dell'Angelico).

Per quanto competa all'Alberti aver riconosciuto il composito (Italicus) come un ordine a sé stante dal capitello avente le stesse proporzioni di quello corinzio (Figure 8,9 ), contrariamente a quanto si è soliti ripetere, non è vero che Vitruvio non ne parli. Egli ne accenna, infatti, a conclusione del primo paragrafo del Libro Quarto aggiungendo che «sed ipsoru(m) vocabula traducta \& co(m)mutata ex corinthiis \& pulvinatis \& doricis videmus $»^{23}$ (Vitr., IV, I, I2).

Si spiega meglio, allora, l'orientamento di Piero verso un composito in cui la porzione ionica e quella corinzia mantengono la loro piena riconoscibilità ${ }^{24}$. Il canale ionico, infatti, è continuo tra una voluta e l'altra, correndo al di sopra dell'echino a ovoli e dardi del labrum del vas corinzio a doppia corona di foglie d'acanto.

23. Cito dall'editio princeps, Firenze, BNC, C.3.25: «Sunt aut(em) hisdem columnis inponunt(ur) capitulor(um) genera variis vocabulis no(m)i(n)ata, quor(um) nec $p($ ro)prietates symmetriar(um) nec colu(m)nar(um) genus aliud no(m)i(n)are possumus, sed ipsoru $(m)$ vocabula traducta \& $c o(m)$ mutata ex corinthiis \& pulvinatis \& doricis videmus. Quor(um) symmetriae sunt in novarum scalpturarum translate subtilitatem».

24. Devono essere le proporzioni corinzie che, ad avviso di Pellegrino Prisciani (Orthopasca: Modena, Biblioteca Estense, Cod. Lat. 466 (alfa.X.1.6), c. 35r) rendono il capitello troppo slanciato: «el capitello de $\mathrm{M}^{\circ}$ Petro dal Borgo qual invero è alto oltre rasonabil mensura». 


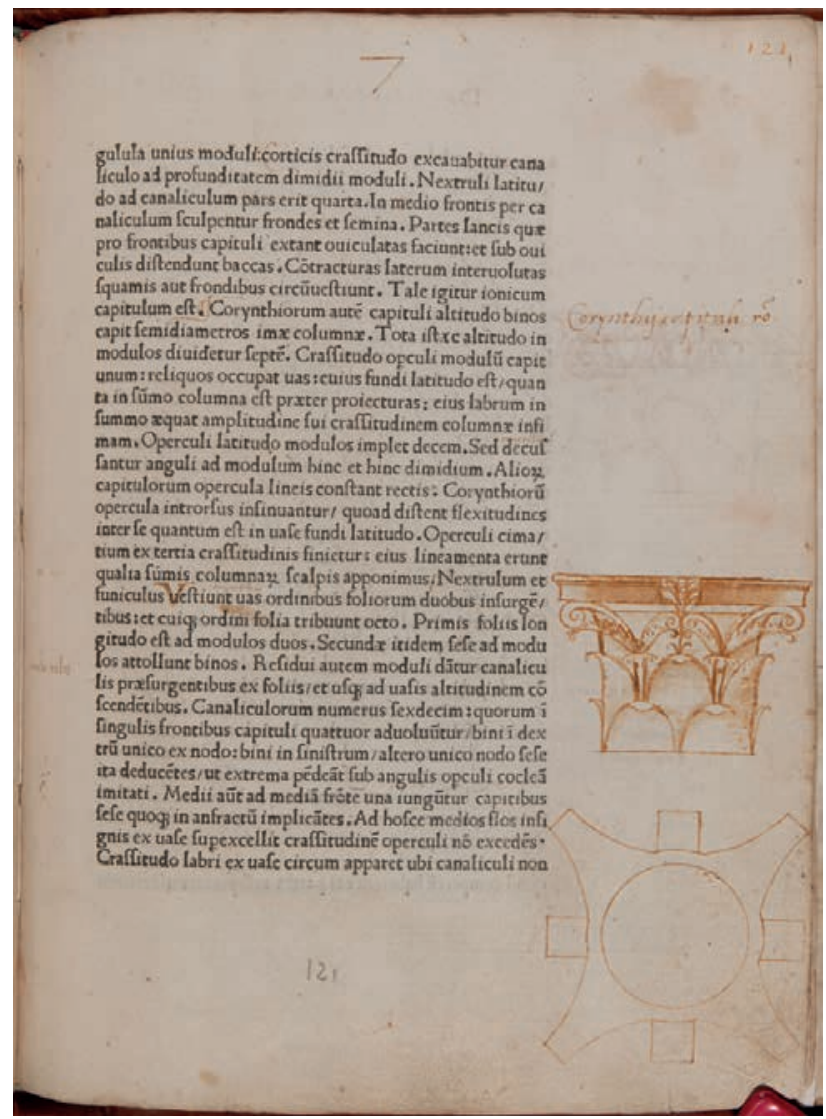

FIGURA 8. PIANTA E PROSPETTO DI UN CAPITELLO CORINZIO IN DOPPIA P.O., IN L.B. ALBERTI, DE RE AEDIFICATORIA, FIRENZE 1485, LIBRO VII, C. I2IR. TORINO, ARCHIVIO ARCIVESCOVILE, S. C.

\section{Anche il capitello composito ${ }^{25}$ è disegnato nella propositio septima del Terzo Libro del De prospectiva pingendi (Figure IO-I2):}

Adunque, $p(e r)$ volere degradare el descripto capitello no(n) lasciando il modo cominciato, farai i(n) propria forma il capitello il quale tu vuoli fare che se vegga da una faccia, del quale fa' ch(e) sia la linea della sua largheça da piè, dove se ferma su la colonna, 4, la quale devidi $p(e r)$ equali in puncto $K$, et sopra $K$ mena la linea $p(e r) p e n d i c u l a r e ~ A K$, che sia 5; poi tira una linea passante $p(e r) A$, equidistante la linea $K$, che sia 7. Poi devidi AK in secte parti equali, delle quali ne poni una delle secte socto $\mathrm{A}$, che sia $\mathrm{AC}$; poi mena la linea equidistante $A$ passante $p(e r) C$, che sia 5 4/7 devisa in $C p(e r)$ equali; poi devidi $A C$ in tre parti equali, delle quali po(n)ne una socto $A$, che sia $B$, et tira la linea eq(ui)distante A passante $p(e r) B$, che sia $611 / 21$, divisa p(er) equali in B; poi piglia il terço de BA et pollo socto $B$, che sia $B$ col tictolo, ch(e) sia el regolecto. Questi sono su la cimasa [...] et hai posti tucti i termini in propria forma su le doi figure. ${ }^{26}$

25. Per la costruzione del capitello composito seguendo il dettato pierfrancescano si veda CAMEROTA, Filippo: «Scheda VI.2», in Camerota, Filippo, Di Teodoro, Francesco Paolo e Grasselli, Luigi (eds.): Op. Cit., pp. 357-358 (Figura 13).

26. La lunga descrizione (qui riprodotta in parte) che si sviluppa per 9 carte, è desunta dal Mss. Regg. A 41/2, cc. 59 r-63r. 


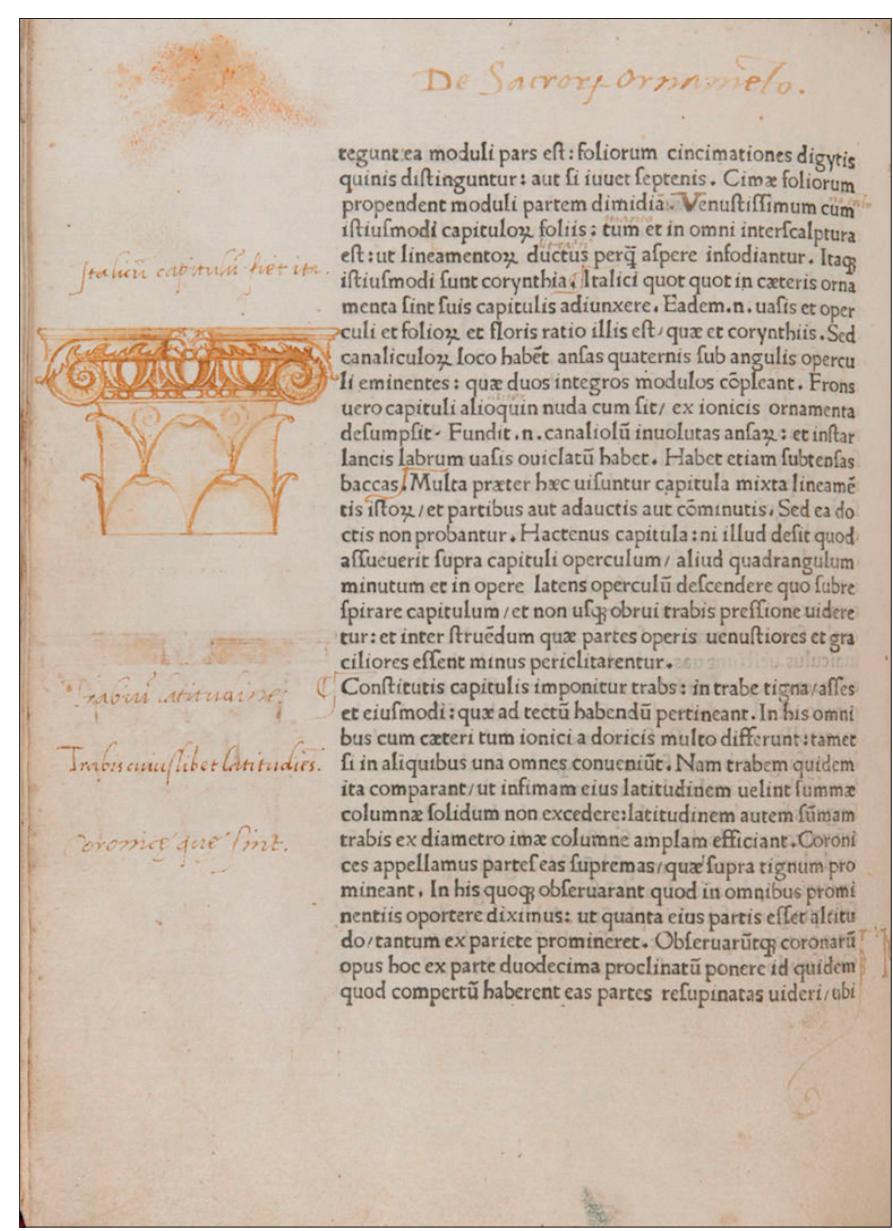

FIGURA 9. PROSPETTO DI UN CAPITELLO COMPOSITO (O «ITALICO»), IN L.B. ALBERTI, DE RE AEDIFICATORIA, FIRENZE 1485, LIBRO VII, C. 121V. TORINO, ARCHIVIO ARCIVESCOVILE, S. C.

Il modo del tracciamento, in pianta e prospetto, e le proporzioni del capitello seguono sia il dettato vitruviano ${ }^{27}$ sia quello albertiano ${ }^{28}$. Né Piero né Alberti, però, propongono i passaggi vitruviani per la definizione delle dimensioni dell'abaco e,

27. «Cori(n)thii generis distribuit r(ati)ones. Eius aut(em) capituli symmetria sic est facie(n)da uti q(uan)ta fuerit crassitudo imae colu(m)nae, tanta sit altitudo capituli cu(m) abaco. Abaci latitudo ita habeat r(ati)o(n)em ut $q($ (uan)ta fuerit altitudo, ta(n)ta duo sint diagonia ab angulo ad angulu(m). Spatia eni(m) ita iustas hebebu(n)t frontes quoquoversus latitudinis frontes sumentur introrsus ab extremis angulis abaci suae frontis latitudinis non ad imu(m) capituli tantam habea(n)t crassitudine(m), q(uan)tam h(ab)et su(m)ma colu(m)na p(rae)ter apothesim \& astragalu(m). Et abaci crassitudo septi(m)a capituli altitudinis. De(m)pta abaci crassitudine, dividat(ur) reliq(ua) pars i(n) partes tres [...]» (Vitr., IV, 1, 11-12). Cito dall'editio princeps, Firenze, BNC, C.3.25.

28. «Coryntiorum aute $(\mathrm{m})$ capituli altitudo binos capit semidiametros imae columnae. Tota istaec altitudo in modulos dividetur septe $(m)$. Crassitudo op(er)culi modulu(m) capit unum, reliquos occupat vas, cuius fundi latitudo est, quanta sit in su(m)mo columna est praeter proiecturas; eius labrum in summo aequat amplitudine sui crassitudinem columnae infimam. Operculi latitudo modulos implet decem; sed decussantur anguli ad modulum hinc et hinc dimidium [...] Operculi cimatium ex tertia crassitudinis finietur; eius lineamenta erunt qualia su(m)mis columnar(um) scalpis apponimus. Nextrulum et funiculus vestiunt vas ordinibus foliorum duobus insurge(n)tibus; et cuiq(ue) ordini folia tribuunt octo. Primis foliis longitudo est ad modulos duos. Secundae itidem sese ad modulos attollunt binos. Residui autem moduli da(n)tur canaliculis praesurgentibus ex foliis et usq(ue) ad vasis altitudinem co(n)scede(n)tibus [...] Italici, quot quot in caeteris ornamenta sint [...]» (De re aed., VII, VIII). Cito dall'editio princeps, Torino, esemplare dell'Archivio arcivescovile, s. collocazione, cc. 121r-121v. 


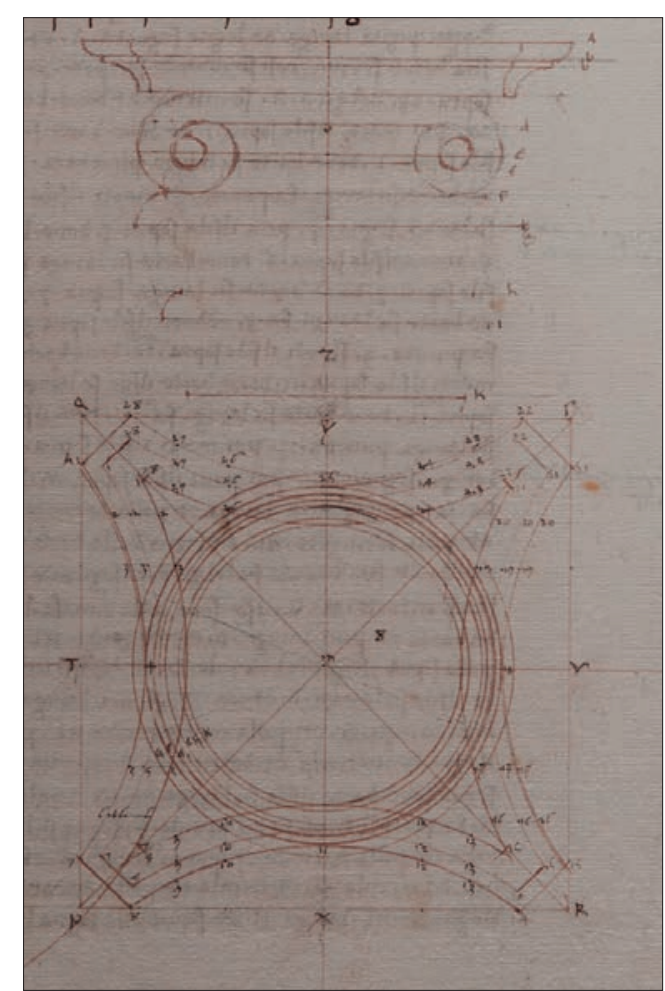

FIGURA 10, PIANTA E PROSPETTO DI UN CAPITELLO COMPOSITO IN DOPPIA P. O. PIERO DELLA FRANCESCA, DE PROSPECTIVA PINGENDI, REGGIO EMILIA, BIBLIOTECA COMUNALE «A. PANIZZI», COD. REGG. A 41/2, C. 63R, PARTICOLARE.

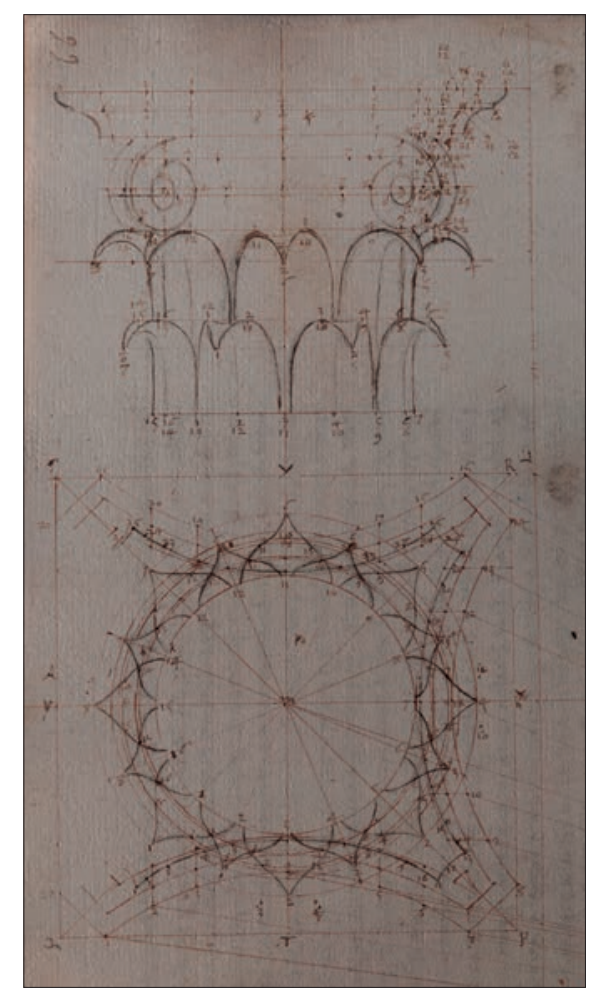

FIGURA 11. PIANTA E PROSPETTO DI UN CAPITELLO COMPOSITO IN DOPPIA P. O. PIERO DELLA FRANCESCA, DE PROSPECTIVA PINGENDI, REGGIO EMILIA, BIBLIOTECA COMUNALE «A. PANIZZI», COD. REGG. A 41/2, C. 66R, PARTICOLARE.

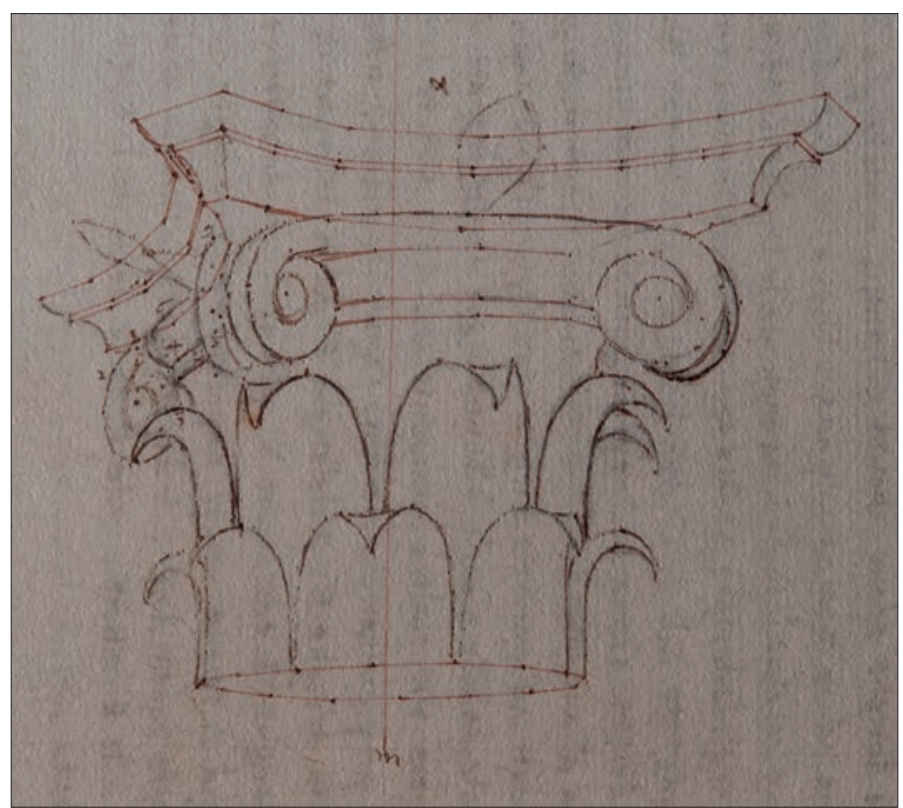

FIGURA 12. PROSPETTIVA DI UN CAPITELLO COMPOSITO. PIERO DELLA FRANCESCA, DE PROSPECTIVA PINGENDI, REGGIO EMILIA, BIBLIOTECA COMUNALE «A. PANIZZI», COD. REGG. A 41/2, C. 67V, PARTICOLARE. 


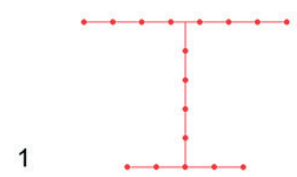

2

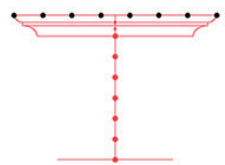

3

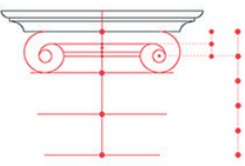

4

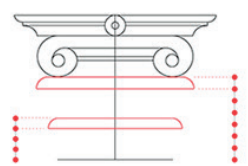

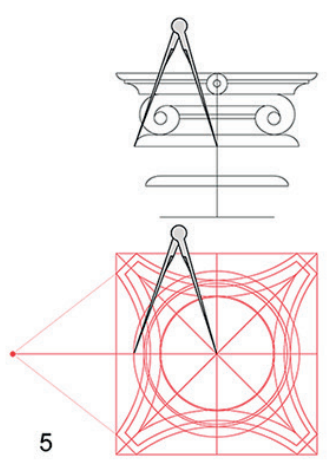
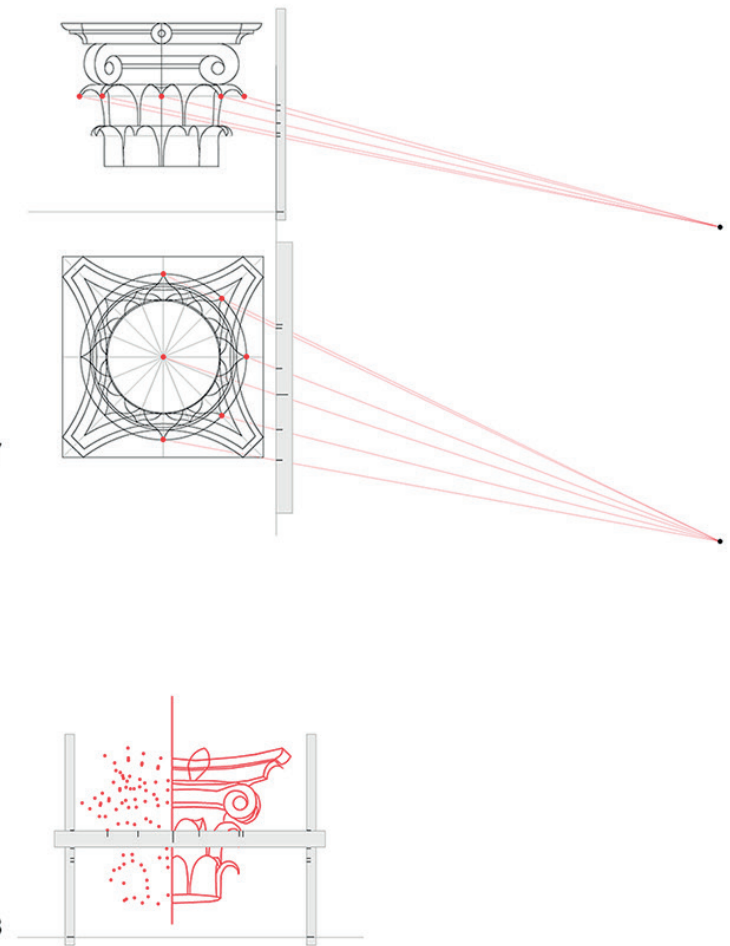

FIGURA 13. COSTRUZIONE PROSPETTICA DI UN CAPITELLO COMPOSITO DA DE PROSPECTIVA PINGENDI (F. CAMEROTA). DA PIERO DELLA FRANCESCA, IL DISEGNO TRA ARTE E SCIENZA...MILANO 2015, P. 224.

soprattutto, non indicano in I/9 del lato del quadrato circoscritto all'abaco il valore della freccia della concavità dei lati curvilinei dell'abaco stesso. Sia Piero sia Alberti partono dalla constatazione che la proiezione della curvatura del lato dell'abaco è tangente al sommoscapo della colonna. È lecito chiedersene la ragione.

Le moderne edizioni critiche e le correnti traduzioni vitruviane riportano tutte la cifra $\mathrm{I} / 9$, ma tale numero frazionario non è rintracciabile in nessun codice del De architectura né nelle tre stampe quattrocentesche dell'opus maius dell'antico trattatista. Il passo era, dunque, corrotto. Spetta, infatti, a Fra Giocondo, averlo emendato per congettura nella sua edizione pubblicata a Venezia nel I5II (Figura I4).

Infatti, lì dove la tradizione manoscritta e le stampe del XV secolo recitano: «latitudinis frontes sumentur introrsus ab extremis angulis abaci suae frontis latitudinis non ad imu(m) capituli tantam habea(n)t crassitudine(m)» $»^{29}$ Giocondo corregge in: «Latitudinis frontes sinuentur introrsus ab extremis angulis abaci, suae fro(n)tis latitudinis nona. Ad imum capituli tantam habeant crassitudinem».

Sia Leon Battista Alberti sia Piero, dunque, hanno dovuto aggirare l'ostacolo.

29. Mio il corsivo. 


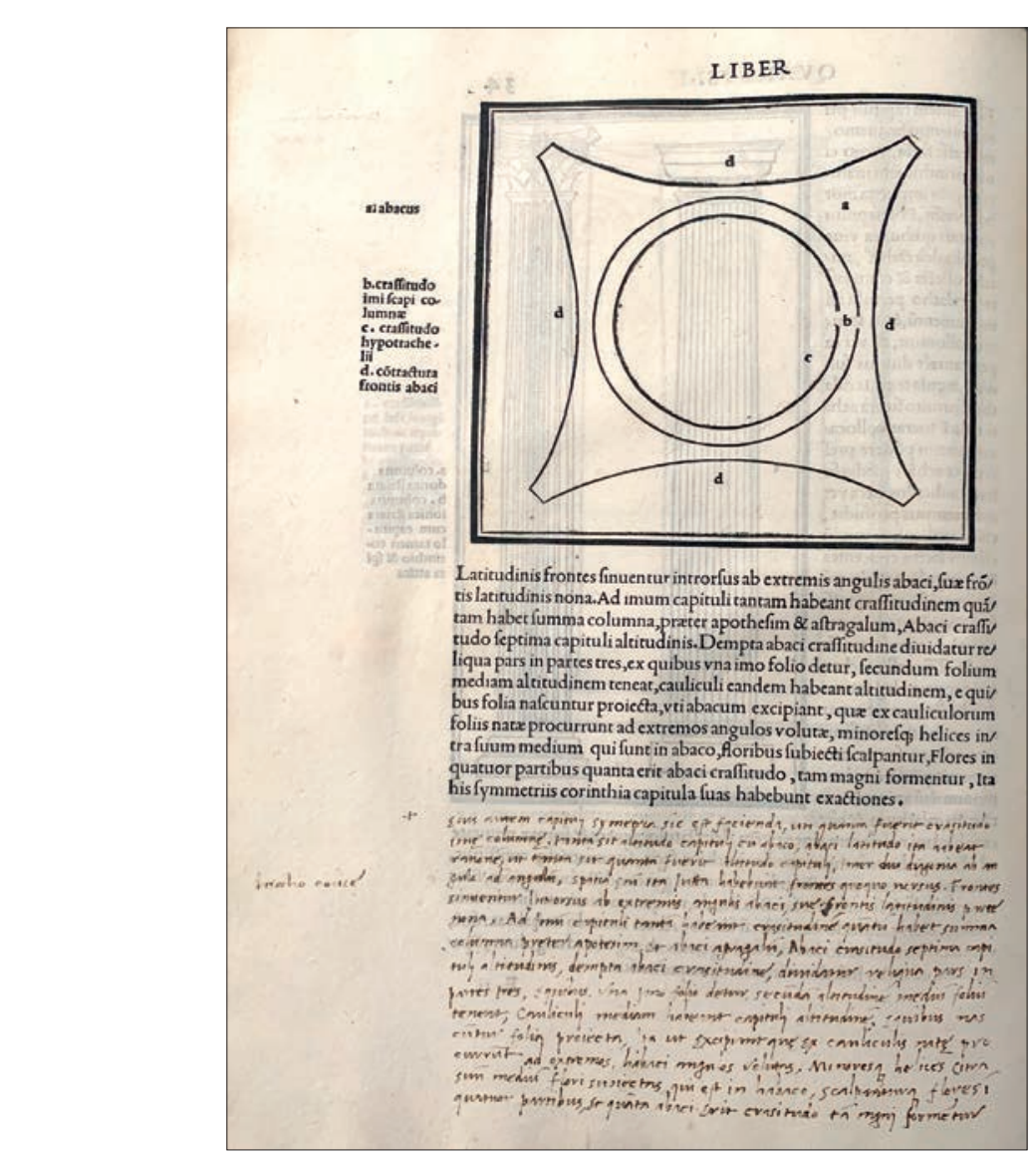

FIGURA 14. PIANTA DI UN CAPITELLO CORINZIO. IN M. VITRUVIUS PER IOCUNDUM ..., VENEZIA 1511, C. 34V.

\section{UN (ENNESIMO) PLAGIO DI PACIOLI}

Anche Luca Pacioli nel suo trattatello d'architettura, poche pagine, appena ventuno, all'interno della Divina proportione ${ }^{30}$, Venezia I509, si occupa sia della base attica sia del capitello composito.

Al pari di Piero della Francesca e di Leon Battista Alberti anche Pacioli, trattando della lunghezza del plinto, dev'essersi trovato di fronte al dilemma tra «sextantem» e «unius \& dimidiae», ma sembra averlo risolto. Egli scrive, infatti, adottando la terminologia albertiana (Figura 15):

La sua basa dev'esser alta la mità del dia(m)etro del suo trochilo inferiore cioè del ef; la qual basa fia co(m)posta de più gradi; che 'I primo ab si chiama da li antichi plinto e da li nostri latastro, qual dev'essere una grosseç̧a e meçça de la colonna longo, con tutto el sportafore o ver proiectura e dev'esser alto el sexto de la grosseça. ${ }^{31}$

30. Cfr. PACIOLI, Luca: Divina proportione. Venezia, per Paganinum de Paganinis de Brixia, 1509, Pars prima [sed Secunda], cc. 23r-33v.

31. Idem, cap. V, c. 28 r. 


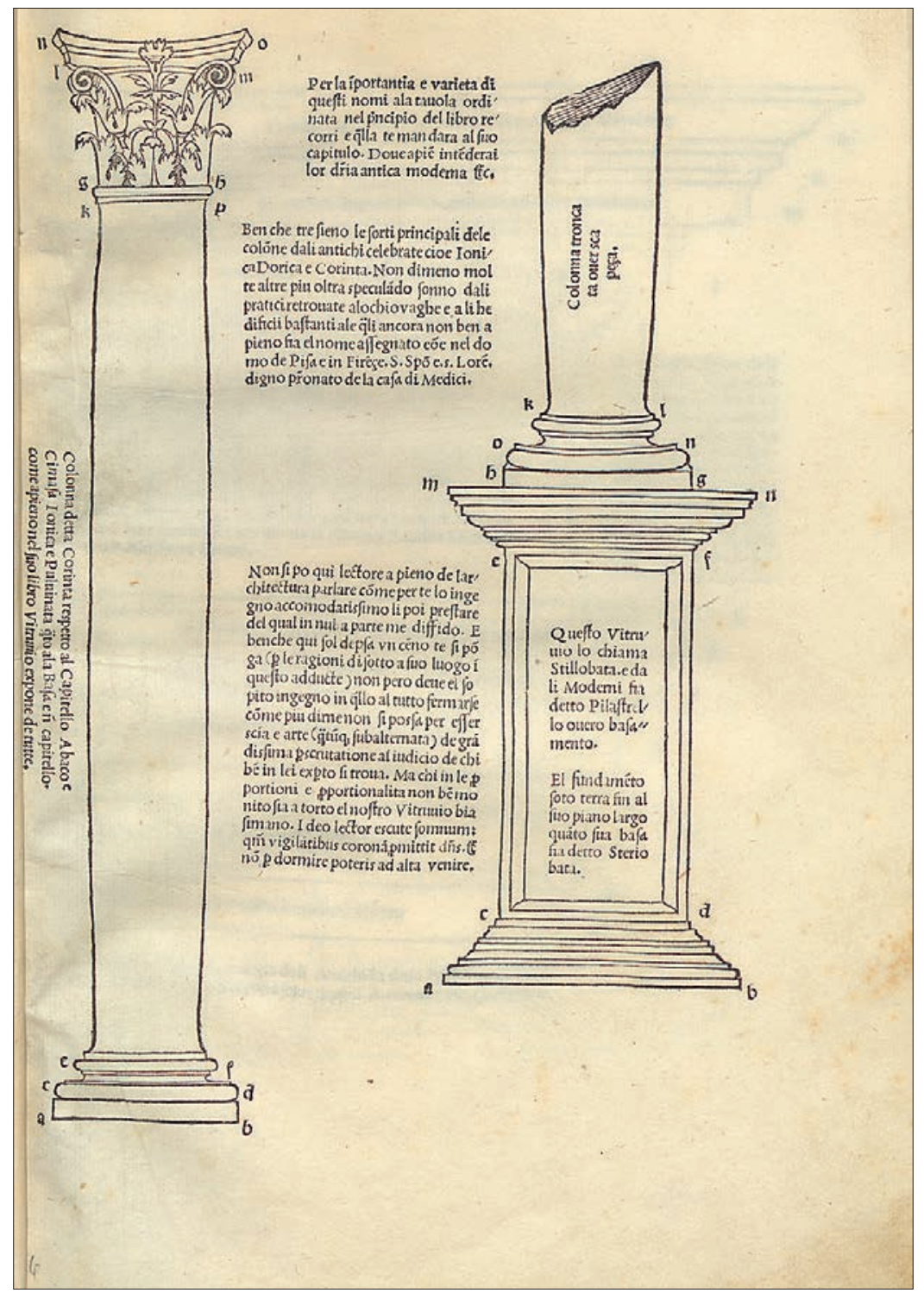

FIGURA 15. ELEMENTI DI UNA COLONNA CORINZIA. DA L. PACIOLI, DE DIVINA PROPORTIONE, VENEZIA 1509, C. N.N.

Come d'incanto Pacioli pare aver risolto anche la questione della curvatura dei lati dell'abaco mistilineo del capitello composito. Pacioli scrive:

[...] in cadauno abaco sonno 4 tetranti. Questi tetranti si formano in questo modo videlicet: se prende el diametro de la contractura de socto cioè de quella gola che posa in su la basa de sotto e quello se dopia e fasse diagonale de un quadrato situato nel cerchio, aponto. E quel tal quadrato, aponto, fia l'abaco de dicto capitello. El suo tetrante se fa cavo verso el centro de dicto quadro o ver tondo, curvandolo el nono de la costa del dicto quadro, cioè curvato fin al sito de l'ochio suo in fronte..$^{32}$

32. Idem, cap. IV, c. 27V. Mio il corsivo. 
Tutte le citazioni latine da Vitruvio nel trattatello di Pacioli - e non sono poche - sono desunte dall'editio florentina, I496 ${ }^{33}$. In ambedue i casi (lunghezza del plinto della base attica e curvatura dell'abaco del capitello composito) il Vitruvio di Pacioli si presenta con la problematicità delle altre due edizioni quattrocentesche (la princeps del I487/I488, l'editio veneta del I497) e dei testimoni manoscritti di cui si è discusso sinora $^{34}$. Stranamente, però, le espressioni usate da Fra Luca sono in volgare e non sono traduzione dei corrispondenti passi dell'edizione fiorentina, che il francescano consultava o possedeva.

La versione italiana di Pacioli presupporrebbe una soluzione filologica del testo latino. Pacioli, però, al pari di Alberti, non era un filologo e non sarebbe stato in grado di dare risposta alcuna ai problemi testuali.

Non resta che una possibilità: i testi di Pacioli sono un plagio da Fra Giocondo.

Il frate veronese, infatti, era il solo che avesse risolto i due problemi: aveva sostituito sestante con quadrante, evitando - seppure non correttamente - la contraddizione nel periodo riguardante l'ह̇кфopá e, per congettura, aveva corretto il passo riguardante la freccia del lato curvilineo dell'abaco composito.

Fin dagli anni Ottanta del Quattrocento Giocondo ${ }^{35}$ lavorava all'edizione del De architectura che vide poi la luce a Venezia nel I5II.

Luca $^{36}$ e Giocondo erano ambedue francescani. Forse si erano conosciuti già a Napoli dove Fra Luca stette dal I488 al I489 e il veronese dal I489 al I49537. Certamente i due ebbero modo di frequentarsi a Venezia nel I508. Fra Giocondo vi risiedeva dal 1506 e vi sarebbe rimasto fino al I5I4; Pacioli vi tenne una memorabile prolusione al commento del quinto libro degli Elementi di Euclide l'in agosto I508, nella chiesa di San Bartolomeo. Tra i presenti, elencati dallo stesso Pacioli nella sua edizione di Euclide, con altri confratelli, notabili e alte personalità delle scienze e

33. Cfr. Di TeOdoro, Francesco Paolo, «Vitruvius in the Trattato dell'Architettura by Luca Pacioli», in MofFAT, Constance and Taglialagamba, Sara (eds.): Illuminating Leonardo. A Festschrift for Carlo Pedretti Celebrating His 70 Years of Scholarschip (1944-2014). Leiden/Boston, Brill, 2016, 114-119.

34. «His perfectis in suis locis spirae collocentur haeq(ue) ad symmetriam sic perficiantur uti crassitudo cum plintho sit. Columnae ex dimidia crassitudine proiecturamq(ue), quam graeci Ecphoran vocant, habeant sextantem; ita tamen lata \& lo(n)ga erit columnae crassitudinis unius \& dimidiae. Altitudo eius si attigurges erit, ita dividatur ut superior pars tertia parte sit crassitudinis columnae, reliquu(m) plintho relinquatur» (Vitr., III, 5, 1-2). «Eius autem capituli symmetria sic est facienda: uti q(ua)nta fuerit crassitudo imae colu(m)nae, tanta sit altitudo capituli $\mathrm{cu}(\mathrm{m})$ abaco. Abaci latitudo ita habeat $r(a t i o) n e m ~ u t ~ q(u a) n t a$ fuerit altitudo, ta(n)ta duo sint diagonia ab angulo ad angulu(m). Spatia eni(m) ita iustas habebu(n)t fro(n)tes quoquoversus latitudinis. Frontes sumentur introrsus ab ectremis angulis abaci suae fro(n)tis latitudinis non ad imu $(m)$ capituli tantam habea(n)t crassitudinem [...]» (Vitr., IV, 1, 11). Cito dall'editio florentina 1496, servendomi dell'esemplare: Firenze, BNC, C.2.11a; miei i corsivi.

35. Per una biografia di Giocondo Cfr. Pagliara, Pier Nicola: «Giovanni Giocondo da Verona (Fra Giocondo)», voce in Dizionario Biografico degli Italiani, LVI, Roma, 2001; la si legga anche in: <http://www.treccani.it/enciclopedia/ giovanni-giocondo-da-verona_(Dizionario-Biografico)/>.

36. Per una biografia del frate matematico di Borgo Sansepolcro Cfr. Dı TeOdoro, Francesco Paolo, «Pacioli, Luca», voce in Dizionario Biografico degli Italiani, LXXX, Roma, 2014; la si legga anche in: <http://www.treccani.it/ enciclopedia/luca-pacioli_(Dizionario-Biografico)/>.

37. La giovinezza di Giocondo si era svolta a Roma ed è certo che avesse relazione con i Riario e i Della Rovere. Certamente Raffaele Riario era un suo mecenate e Giocondo aveva avuto accesso al palazzo dei Santissimi Apostoli. Fu Giulio II a chiedergli nel 1505 o nel 1506 un progetto per la nuova basilica di San Pietro. Con la stessa famiglia dei papi Sisto IV e Giulio II aveva rapporti Pacioli. Fu, infatti, nel palazzo dell'allora cardinale Giuliano della Rovere che Fra Luca mostrò a Guidubaldo da Montefeltro alcuni modelli (le «forme materiali») dei cinque poliedri regolari con «molti altri da ditti regulari dependenti».

${ }_{33}$ Cfr. Euclidis megarensis ... op(er)a... Lucas Paciolus ... detersit. Venezia, 1509, c. 31r. 
delle arti compariva anche «Frater locundus Veronensis Antiquarius» nella sezione «Reverendi Sacre Theologie Professores» ${ }^{33}$. A Venezia Pacioli dovette avere accesso ai materiali di Giocondo discutendo con lui di architettura e di Vitruvio mentre era intento alla pubblicazione degli Elementa di Euclide e della Divina proportione stampati da Paganino de' Paganini nel $1509^{38}$.

In quest'ultima opera fece "suoi» ${ }^{99}$ alcuni risultati del faticoso e più che trentennale impegno profuso da Fra Giovanni Giocondo nello studio, nell'insegnamento e nell'emendatio del De architectura, consultando e cercando codici tra l'Italia, percorsa in lungo e in largo, e la Francia di Carlo VIIl e Luigi XII ${ }^{40}$.

38. È ipotizzabile anche una non breve consuetudine tra i due francescani per l'intermediario di Giovan Marco Canozi, figlio di Lorenzo Canozi da Lendinara, amico fraterno di Piero della Francesca. Giovan Marco, che lavorava per la Repubblica veneta nei medesimi anni in cui lo stesso Giocondo vi era all'opera, viene ricordato nella prefazione al trattatello di architettura come «Giovanmarco mio caro co(m)pare», architetto e cavatore di canali a Venezia. Lo stesso Canozi è rammentato tra i presenti in San Bartolomeo come «loa(n)nes Marcus Canotius Patavinus», incluso nell'elenco dei «Medici Illustres».

39. «Stranamente» quando traduce Pacioli non sente di dover rendere conto del nome dell'autore. Aveva fatto così anche con il volgarizzamento del Libellus de quinque corporibus regularibus di Piero della Francesca, pubblicato nella Divina proporzione con il titolo Libellus in tres partiales divisus q(ui)nq(ue) corporu $(m)$ regularium et depe $(n) \operatorname{dentiu}(m)$ con dedica a Pier Soderini.

40. Sul frate architetto veronese si veda ora Gros, Pierre e PAGLIARA, Pier Nicola (eds.): Giovanni Giocondo umanista, architetto e antiquario. Venezia, Marsilio, 2014. 


\section{BIBLIOGRAFIA}

Alberti, Leon Battista: De re aedificatoria. Firenze, Niccolò di Lorenzo Alamanni, I485.

Basto, João: «The composite capitals in Piero della Francesca's Flagellation», in DAlAI Emiliani, Marisa e Curzi, Valter (eds.): Piero della Francesca tra arte e scienza, Atti del convegno internazionale di studi (Arezzo, 8-II ottobre I992, Sansepolcro, I2 ottobre I992) Venezia, Marsilio, I996, 77-94.

Bertolini, Lucia: «Appunti su cronologia e circolazione del De prospectiva pingendi», in Camerota, Filippo e Di Teodoro, Francesco Paolo (eds.): Piero della Francesca, la seduzione della prospettiva, catalogo della mostra (Sansepolcro, 2I marzo20I8-6 gennaio 20I9). Venezia, Marsilio, 20I8, 25-3I.

Camerota, Filippo: «Scheda VI.I», in Camerota, Filippo, Di Teodoro, Francesco Paolo e Grasselli, Luigi (eds.): Piero della Francesca, il disegno tra arte e scienza, cat. della mostra (Reggio Emilia I4 marzo-I3 giugno 20I5). Milano, Skira, 20I5, 356-357.

Camerota, Filippo, «Scheda VI.2», in Camerota, Filippo, Di Teodoro, Francesco Paolo e Grasselli, Luigi (eds.): Piero della Francesca, il disegno tra arte e scienza, cat. della mostra (Reggio Emilia I4 marzo-I3 giugno 20I5). Milano, Skira, 2015, 357-358.

DAly DAvis, Margaret: Piero della Francesca's Mathematical Treatises, The «Trattato d'abaco» and «Libellus de quinque corporibus regularibus». Ravenna, Longo, I977.

Di Teodoro, Francesco Paolo: «L'architettura della Trinità», in Camerota, Filippo (ed.): Nel segno di Masaccio. L'invenzione della prospettiva, cat. della mostra (Firenze I6 ottobre 200I-20 gennaio 2002). Firenze, Giunti, 200I, 47-5I.

Di Teodoro, Francesco Paolo: «Intorno a una princeps del De re aedificatoria», in CALzonA, Arturo et alii: Leon Battista Alberti, architetture e committenti, atti dei convegni internazionali (Firenze, Rimini, Mantova, I2-I6 ottobre 2004), Il. Firenze, Olschki, 2009, 589-6I5.

Di Teodoro, Francesco Paolo: «Marmorea templa»: Firenze, identità romana e tutela identitaria», in Burns, H, Mussolin, Mauro (eds.), con la collaborazione di Altavista, Clara: Architettura e identità locali, Il. Firenze, Olschki, 20I3, 449-469.

Di Teodoro, Francesco Paolo: ««... che pare sia bucato quel muro»: l'architettura verosimile della Trinità di Masaccio», in CAzzato, Vincenzo, Roberto, Sebastiano e Bevilacqua, Mario (eds.): La festa delle arti. Scritti in onore di Marcello Fagiolo per i cinquant'anni di studi, I. Roma, Gangemi, 20I4, 206-2II.

Di Teodoro, Francesco Paolo: «Pacioli, Luca», voce in Dizionario Biografico degli Italiani, LXXX, Roma, 2014.

Di TeOdoro, Francesco Paolo, «Vitruvius in the Trattato dell'Architettura by Luca Pacioli», in Moffat, Constance and Taglialagamba, Sara (eds.): Illuminating Leonardo. A Festschrift for Carlo Pedretti Celebrating His 70 Years of Scholarschip (1944-20I4). Leiden/Boston, Brill, 20I6, II4-II9.

Euclidis megarensis ... op(er)a... Lucas Paciolus ... detersit. Venezia, I509.

Giocondo, Giovanni (ed.): M. Vitruvius ... solito castigatior factus cum figuris. Venezia, Ioannis de Tridino alias Tacuino, I5II.

Gros, Pierre e Pagliara, Pier Nicola (eds.): Giovanni Giocondo umanista, architetto e antiquario. Venezia, Marsilio, 2014.

PACiOlı, Luca: Divina proportione. Venezia, per Paganinum de Paganinis de Brixia, I509.

Pagliara, Pier Nicola: «Giovanni Giocondo da Verona (Fra Giocondo)», voce in Dizionario Biografico degli Italiani, LVI, Roma $200 \mathrm{I}$. 
Piero Della Francesca: De prospectiva pingendi. Gizzi, Chiara (ed.). Venezia, Edizioni Ca' Foscari, 2016.

Vitruvıus: De architectura libri decem. KROHN, Fritz (ed.). Leipzig, Teubner, I9I2 («Bibliotheca scriptorum Graecorum et Romanorum Teubneriana»). 



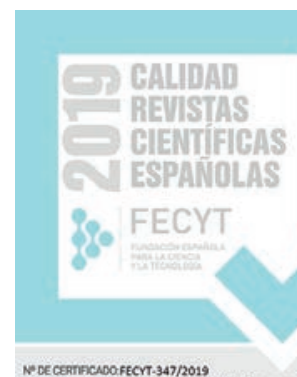

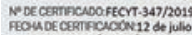

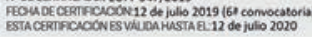

\section{SERIE VII HISTORIA DEL ARTE}

REVISTA DE LA FACULTAD DE GEOGRAFÍA E HISTORIA
AÑO 2019

NUEVA ÉPOCA

ISSN: $1130-4715$

E-ISSN 2340-1478

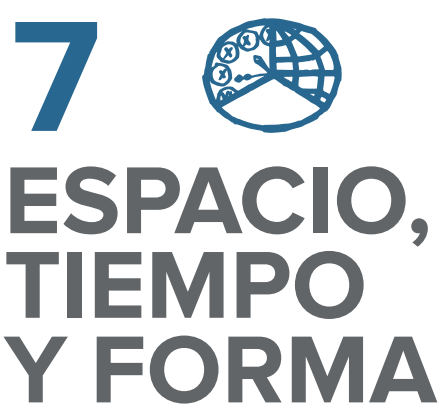

Dossier per Filippo Camerota: L'Apelle Vitruviano: Riflessioni sulla cultura architettonica dei pittori nella prima età moderna - El Apelles vitruviano: Reflexiones en torno a la cultura arquitectónica de los pintores de la Edad Moderna

17 FILIPPO CAMEROTA (GUEST EDITOR)

Introduzione. La prospettiva come tema vitruviano · Introduction.

Perspective as a Vitruvian Theme

4.1 Francesco P. Di TeOdoro (GUest author)

Due quæstiones vitruviane riconosciute: la base attica e il capitello composito nel terzo libro del De prospectiva pingendi di Piero della Francesca e un plagio conclamato di Luca Pacioli . Two Recognized Vitruvian Problems: The Attic Base and the Composite Capital in the Third Book of De Prospectiva Pingendi by Piero della Francesca and an Evident Plagiarism by Luca Pacioli

65 GIOVANNI MARIA FARA (GUEST AUTHOR) and Vitruvius

77 CARMEn GONZÁlez-RomÁn

Metaescenografías pintadas · Painted Meta-scenographies

103 SARA FuENTES LÁZARo

Ad vitandam confusionem. Una aproximación analítica al tratado sobre perspectiva de Andrea Pozzo . Ad vitandam confusionem. An Analytical Approach to Andrea Pozzo's Treatise on Perspective

\section{Miscelánea · Miscellany}

133 ANTONIO PÉREZ LARGACHA

Art in Egypt. Ritual, Sense and Function

Predynastic

161 Alejandra IzQuierdo Perales

contexto de los templos ptolemaicos. The Temple of Hathor in Deir el-Medina: An Iconographic Study in the Context of the Ptolemaic Temples

191 JaIme Moraleda Moraleda

The Work of Miniatures for the Book of Vows of the City Hall of Toledo
209 Sergio Ramírez González, antonio Bravo nieto \& Juan ANTONIO BELLVER GARRIDO

La recuperación de dos repuestos de pólvora del siglo XVIII en Melilla: análisis y restauración - Recovery of Two Spare Gunpowder Warehouses from the XVIII ${ }^{\text {th }}$ Century in Melilla: Analysis and Restoration

231 Alejandro de LA Fuente Escribano

expresión del romanticismo en España . The Restoration of Guadamur Castle in the XIX $X^{\text {th }}$ Century as an Expression of Romanticism in Spain

26 Paula Gabriela núñez, Carolina lema, Carolina Michel \& MAIA VARGAS

La construcción estatal patagónica en el siglo XIX. El dibujo como arte científico $€$ institucional . The Patagonian State Construction in XIX ${ }^{\text {th }}$ Century. The Drawing as Scientific and Institutional Art

\section{Guillermo Juberías Gracia} la zarzuela Pan y Toros (1864) en las colecciones municipales de Madrid · A Nineteenth-Century Vision of Charles IV Spain: Designs for the Zarzuela Pan $y$ Toros (1864) in the Municipal Collections of Madrid

311 Aurora Fernández Polanco

Ojos curiosos y capital: sobre el turismo visual decimonónico · Curious Eyes and Capital: About Nineteenth-Century Visual Tourism

327 AngÉlica García-Manso

Los cinematógrafos diseñados por Fernando Perianes: una lectura Movie-theaters Planned by Fernando Perianes: A Heritage Reading around Leisure Architecture in the Province of Cáceres (Spain)

361 José-Carlos Delgado Gómez

y el médico y caricaturista José Delgado Úbeda «Zas» . The Humoristic Halls' during the Spanish Postwar Period (1940-1953) and the Doctor and Caricaturist José Delgado Úbeda «Zas»

379 IOANNIS MOURATIDIS

The Space Dimension of «Being A Museum User»: Reflections on the Social Construction of an Inclusive Exhibition Space

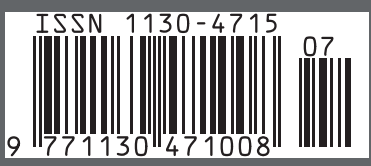


AÑO 2019

NUEVA ÉPOCA

ISSN: 1130-4715

E-ISSN 2340-1478

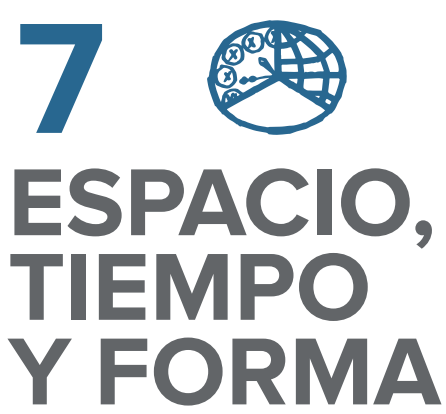

Reseñas · Book Reviews

4.07 Julia Fernández Toledano arte y la política en los tiempos del telón de acero. Madrid, Brumaria, 2019

\subsection{M. ${ }^{a}$ Cristina Hernández Castelló}

Leonardo da Vinci. Munich, Himer Publishers, 2018

\section{Francisco ORTS-RUIZ}

Mínguez, Víctor (dir.), El linaje del rey monje. La configuración cultural e iconográfica de la Corona aragonensis (1164-1516). Castelló de la Plana, Publicacions de la Universitat Jaume I, 2018. 